\title{
Minimal wave speed in a dispersal predator-prey system with delays
}

\section{Xue-Shi Li ${ }^{1 *}$, Shuxia Pan ${ }^{2}$ and Hong-Bo Shi ${ }^{3}$}

*Correspondence: lixuesh@yeah.net; lixueshi0925@126.com

'School of Mathematics, Lanzhou City University, Lanzhou, People's Republic of China Full list of author information is available at the end of the article

\begin{abstract}
This paper is concerned with the minimal wave speed in a nonlocal dispersal predator-prey system with delays. We define a threshold. By presenting the existence and nonexistence of traveling wave solutions, we confirm that the threshold is the minimal wave speed, which completes the known results.
\end{abstract}

Keywords: Upper-lower solutions; Asymptotic spreading; Contracting rectangle; Nonmonotone system

\section{Introduction}

Spatial propagation dynamics of parabolic type systems has been widely investigated in the literature. In the past decades, some important results were established for monotone semiflows; see [1-6] and a survey paper by Zhao [7]. In particular, there are some important thresholds that have been widely and intensively studied, and one is the minimal wave speed of traveling wave solutions, which plays an important role modeling biological processes and chemical kinetic $[8,9]$. Here, the minimal wave speed implies the existence (nonexistence) of a desired traveling wave solution if the wave speed is not less (is less) than the threshold.

It is well known that energy transfer is one basic law in nature and one typical model on the topic is the predator-prey system, and the spatial distribution of individuals is also important to understand the evolutionary process [10-13]. Since the work of Dunbar [1416], much attention has been paid to traveling wave solutions of reaction-diffusion systems with predator-prey nonlinearities to model the transmission of energy. However, the dynamics of predator-prey systems is a very field of research since they do not generate monotone semiflows, and there are many open problems on the minimal wave speed of traveling wave solutions.

In this paper, we shall investigate the following nonmonotone system:

$$
\left\{\begin{array}{l}
\frac{\partial u_{1}(x, t)}{\partial t}=d_{1}\left[J_{1} * u_{1}\right](x, t)+r_{1} u_{1}(x, t) F_{1}\left(u_{1}, u_{2}\right)(x, t), \\
\frac{\partial u_{2}(x, t)}{\partial t}=d_{2}\left[J_{2} * u_{2}\right](x, t)+r_{2} u_{2}(x, t) F_{2}\left(u_{1}, u_{2}\right)(x, t),
\end{array}\right.
$$


in which $x \in \mathbb{R}, t>0,\left(u_{1}, u_{2}\right) \in \mathbb{R}^{2}, r_{1}, r_{2}, d_{1}$ and $d_{2}$ are positive constants, $F_{1}$ and $F_{2}$ are defined by

$$
\begin{aligned}
F_{1}\left(u_{1}, u_{2}\right)(x, t)= & 1-a_{1} u_{1}(x, t) \\
& -b_{1} \int_{-\tau}^{0} u_{1}(x, t+s) d \eta_{11}(s)-c_{1} \int_{-\tau}^{0} u_{2}(x, t+s) d \eta_{12}(s), \\
F_{2}\left(u_{1}, u_{2}\right)(x, t)= & 1-a_{2} u_{2}(x, t) \\
& -b_{2} \int_{-\tau}^{0} u_{2}(x, t+s) d \eta_{22}(s)+c_{2} \int_{-\tau}^{0} u_{1}(x, t+s) d \eta_{21}(s),
\end{aligned}
$$

hereafter, $a_{1}>0, a_{2}>0, b_{1} \geq 0, b_{2} \geq 0, c_{1} \geq 0, c_{2} \geq 0, \tau>0$ are constants such that

$$
\eta_{i j}(s) \text { is nondecreasing on }[-\tau, 0] \quad \text { and } \quad \eta_{i j}(0)-\eta_{i j}(-\tau)=1, \quad i, j=1,2 \text {. }
$$

Moreover, $\left[J_{1} * u_{1}\right](x, t)$ and $\left[J_{2} * u_{2}\right](x, t)$ formulate the spatial dispersal of individuals (see Bates [17], Fife [18] and Hopf [19] for the backgrounds and applications of dispersal models) and are illustrated by

$$
\begin{aligned}
& {\left[J_{1} * u_{1}\right](x, t)=\int_{\mathbb{R}} J_{1}(x-y)\left[u_{1}(y, t)-u_{1}(x, t)\right] d y,} \\
& {\left[J_{2} * u_{2}\right](x, t)=\int_{\mathbb{R}} J_{2}(x-y)\left[u_{2}(y, t)-u_{2}(x, t)\right] d y,}
\end{aligned}
$$

where $J_{1}, J_{2}$ are probability kernel functions formulating the random dispersal of individuals and satisfy the following assumptions:

(J1) $J_{i}$ is nonnegative and continuous for each $i=1,2$;

(J2) for any $\lambda \in \mathbb{R}, \int_{\mathbb{R}} J_{i}(y) e^{\lambda y} d y<\infty, i=1,2$;

(J3) $\int_{\mathbb{R}} J_{i}(y) d y=1, J_{i}(y)=J_{i}(-y), y \in \mathbb{R}, i=1,2$.

Clearly, (1.1) is a predator-prey system and does not generate monotone semiflows. In Yu and Yuan [20], Zhang et al. [21], if $a_{1}=a_{2}=0$ with small delay or $b_{1}=b_{2}=0$, the authors obtained a threshold. If the wave speed is larger than the threshold, they proved the existence of traveling wave solutions, which formulates that both the predator and the prey invade a new habitat. But the question remains open of the existence or nonexistence of traveling wave solution if the wave speed is not larger than the threshold. Our main purpose of this paper is to answer the question.

The rest of this paper is organized as follows. In Sect. 2, we recall some known results. Section 3 is concerned with the existence of nonconstant traveling wave solutions. In Sect. 4, the asymptotic behavior and nonexistence of traveling wave solutions are presented. Finally, we give a discussion of the methods and results in this paper.

\section{Preliminaries}

In this part, we shall give some preliminaries. Since $a_{1}>0, a_{2}>0$ are positive constants, we assume that $a_{1}=a_{2}=1$ due to the scaling recipe. Let

$$
\left(u_{1}(x, t), u_{2}(x, t)\right)=\left(\phi_{1}(\xi), \phi_{2}(\xi)\right), \quad \xi=x+c t,
$$


be a traveling wave solution of (1.1). Then $\left(\phi_{1}(\xi), \phi_{2}(\xi)\right)$ and $c$ satisfy

$$
\begin{cases}d_{1}\left[J_{1} * \phi_{1}\right](\xi)-c \phi_{1}^{\prime}(\xi)+r_{1} \phi_{1}(\xi) F_{1}\left(\phi_{1}, \phi_{2}\right)(\xi)=0, & \xi \in \mathbb{R}, \\ d_{2}\left[J_{2} * \phi_{2}\right](\xi)-c \phi_{2}^{\prime}(\xi)+r_{2} \phi_{2}(\xi) F_{2}\left(\phi_{1}, \phi_{2}\right)(\xi)=0, & \xi \in \mathbb{R}\end{cases}
$$

with

$$
\begin{aligned}
& \left.J_{1} * \phi_{1}\right](\xi)=\int_{\mathbb{R}} J_{1}(y) \phi_{1}(\xi-y) d y-\phi_{1}(\xi), \\
& \left.J_{2} * \phi_{21}\right](\xi)=\int_{\mathbb{R}} J_{2}(y) \phi_{2}(\xi-y) d y-\phi_{2}(\xi),
\end{aligned}
$$

and

$$
\begin{aligned}
F_{1}\left(\phi_{1}, \phi_{2}\right)(\xi)= & 1-\phi_{1}(\xi) \\
& -b_{1} \int_{-\tau}^{0} \phi_{1}(\xi+c s) d \eta_{11}(s)-c_{1} \int_{-\tau}^{0} \phi_{2}(\xi+c s) d \eta_{12}(s), \\
F_{2}\left(\phi_{1}, \phi_{2}\right)(\xi)= & -\phi_{2}(\xi) \\
& -b_{2} \int_{-\tau}^{0} \phi_{2}(\xi+c s) d \eta_{22}(s)+c_{2} \int_{-\tau}^{0} \phi_{1}(\xi+c s) d \eta_{21}(s) .
\end{aligned}
$$

Similar to [20,22], we shall focus on the positive $\left(\phi_{1}, \phi_{2}\right)$ satisfying

$$
\lim _{\xi \rightarrow-\infty} \phi_{i}(\xi)=0, \quad \lim _{\xi \rightarrow \infty} \phi_{i}(\xi)=k_{i}, \quad i=1,2,
$$

where $\left(k_{1}, k_{2}\right)$ is the unique spatial homogeneous steady state of $(1.1)$ and

$$
k_{1}=\frac{1+b_{2}-c_{1}}{\left(1+b_{1}\right)\left(1+b_{2}\right)+c_{1} c_{2}}, \quad k_{2}=\frac{1+b_{1}+c_{2}}{\left(1+b_{1}\right)\left(1+b_{2}\right)+c_{1} c_{2}}
$$

provided that

$$
1+b_{2}>c_{1} \text {. }
$$

When the scalar equation is concerned, Jin and Zhao [23] studied a periodic equation with dispersal. Their results remain true for the following equation with constant coefficients:

$$
\left\{\begin{array}{l}
\frac{\partial u(x, t)}{\partial t}=d[J * u](x, t)+r u(x, t)[1-u(x, t)] \\
u(x, 0)=\chi(x), \quad x \in \mathbb{R}
\end{array}\right.
$$

where $J$ satisfies (J1)-(J3), $d>0$ and $r>0$ are constants, and the initial value $\chi(x)$ is uniformly continuous and bounded. By [23], Theorem 2.3, we have the following comparison principle of (2.4). 
Lemma 2.1 Assume that $0 \leq \chi(x) \leq 1$. Then (2.4) admits a solution for all $x \in \mathbb{R}, t>0$. If $w(x, 0)$ is uniformly continuous and bounded, and $w(x, t)$ satisfies

$$
\left\{\begin{array}{l}
\frac{\partial w(x, t)}{\partial t} \geq(\leq) d[J * w](x, t)+r w(x, t)[1-w(x, t)], \quad x \in \mathbb{R}, t>0 \\
w(x, 0) \geq(\leq) \chi(x), \quad x \in \mathbb{R}
\end{array}\right.
$$

then

$$
w(x, t) \geq(\leq) u(x, t), \quad x \in \mathbb{R}, t>0 .
$$

For $\lambda>0$, define

$$
c^{\prime}=\inf _{\lambda>0} \frac{d\left[\int_{\mathbb{R}} J(y) e^{\lambda y} d y-1\right]+r}{\lambda} .
$$

Then $c^{\prime}>0$ holds. Moreover, it also admits the following property [23].

Lemma 2.2 Assume that $\chi(x)>0$. Then, for any $c<c^{\prime}$, we have

$$
\liminf _{t \rightarrow \infty} \inf _{|x|<c t} u(x, t)=\limsup _{t \rightarrow \infty} \sup _{|x|<c t} u(x, t)=1 .
$$

If $\chi(x)$ has nonempty compact support, then

$$
\lim _{t \rightarrow \infty} \sup _{|x|>c t} u(x, t)=0, \quad c>c^{\prime} .
$$

For $\lambda>0, c>0$, we further define $c^{*}=\max \left\{c_{1}^{*}, c_{2}^{*}\right\}$ with

$$
\begin{aligned}
& c_{1}^{*}=\inf _{\lambda>0} \frac{d_{1}\left[\int_{\mathbb{R}} J_{1}(y) e^{\lambda y} d y-1\right]+r_{1}}{\lambda}, \\
& c_{2}^{*}=\inf _{\lambda>0} \frac{d_{2}\left[\int_{\mathbb{R}} J_{2}(y) e^{\lambda y} d y-1\right]+r_{2}}{\lambda},
\end{aligned}
$$

and

$$
\begin{aligned}
& \Theta_{1}(\lambda, c)=d_{1}\left[\int_{\mathbb{R}} J_{1}(y) e^{\lambda y} d y-1\right]-c \lambda+r_{1}, \\
& \Theta_{2}(\lambda, c)=d_{2}\left[\int_{\mathbb{R}} J_{2}(y) e^{\lambda y} d y-1\right]-c \lambda+r_{2} .
\end{aligned}
$$

By the convexity, we have the following conclusion.

Lemma 2.3 Assume that $c^{*}, \Theta_{1}(\lambda, c), \Theta_{2}(\lambda, c)$ are defined as the above.

(1) $c_{i}^{*}>0$ holds and $\Theta_{i}(\lambda, c)=0$ has two distinct positive roots $\lambda_{i}^{c}<\lambda_{i+2}^{c}$ for any $c>c^{*}$ and each $i=1,2$. Moreover, for each $i=1,2$, and $c>c_{i}^{*}$, if $\lambda_{i} \in\left(\lambda_{i}^{c}, \lambda_{i+2}^{c}\right)$, then $\Theta_{i}\left(\lambda_{i}, c\right)<0$.

(2) If $c \in\left(0, c_{i}^{*}\right)$, then $\Theta_{i}(\lambda, c)>0$ for any $\lambda>0$ and $i=1,2$.

(3) If $c=c_{i}^{*}$, then $\Theta_{i}\left(\lambda, c^{*}\right) \geq 0$ for any $\lambda>0$ and $\Theta_{i}\left(\lambda, c^{*}\right)=0$ has a unique positive root $\lambda_{i}^{*}$, where $i=1,2$. 
For convenience, we use the following notation:

$$
\begin{aligned}
H_{1}\left(\phi_{1}, \psi_{1}, \phi_{2}\right)(\xi)= & 1-\phi_{1}(\xi) \\
& -b_{1} \int_{-\tau}^{0} \psi_{1}(\xi+c s) d \eta_{11}(s)-c_{1} \int_{-\tau}^{0} \phi_{2}(\xi+c s) d \eta_{12}(s), \\
H_{2}\left(\phi_{1}, \psi_{1}, \phi_{2}\right)(\xi)= & 1-\phi_{2}(\xi) \\
& -b_{2} \int_{-\tau}^{0} \psi_{1}(\xi+c s) d \eta_{22}(s)+c_{2} \int_{-\tau}^{0} \phi_{1}(\xi+c s) d \eta_{21}(s),
\end{aligned}
$$

for any positive bounded continuous functions $\phi_{1}(\xi), \psi_{1}(\xi), \phi_{2}(\xi), \xi \in \mathbb{R}$.

Similar to Pan [24], Theorem 3.2, we can prove the following conclusions.

Lemma 2.4 Assume that $\underline{\phi}_{1}(\xi), \bar{\phi}_{1}(\xi), \underline{\phi}_{2}(\xi), \bar{\phi}_{2}(\xi)$ are continuous functions satisfying

(A1) $0 \leq \underline{\phi}_{1}(\xi) \leq \bar{\phi}_{1}(\xi) \leq 1,0 \leq \underline{\phi}_{2}(\xi) \leq \bar{\phi}_{2}(\xi) \leq 1+c_{2}, \xi \in \mathbb{R}$;

(A2) there exists a set $E$ containing finite points of $\mathbb{R}$ such that they are differentiable and their derivatives are bounded if $\xi \in \mathbb{R} \backslash E$;

(A3) they satisfies the following inequalities:

$$
\begin{aligned}
& d_{1}\left[J_{1} * \bar{\phi}_{1}\right](\xi)-c \bar{\phi}_{1}^{\prime}(\xi)+r_{1} \bar{\phi}_{1}(\xi) H_{1}\left(\bar{\phi}_{1}, \underline{\phi}_{1}, \underline{\phi}_{2}\right)(\xi) \leq 0, \\
& d_{1}\left[J_{1} * \underline{\phi}_{1}\right](\xi)-c \underline{\phi}_{1}^{\prime}(\xi)+r_{1} \underline{\phi}_{1}(\xi) H_{1}\left(\underline{\phi}_{1}, \bar{\phi}_{1}, \bar{\phi}_{2}\right)(\xi) \geq 0, \\
& \left.d_{2} J_{2} * \bar{\phi}_{2}\right](\xi)-c \bar{\phi}_{2}^{\prime}(\xi)+r_{2} \bar{\phi}_{2}(\xi) H_{2}\left(\bar{\phi}_{1}, \underline{\phi}_{2}, \bar{\phi}_{2}\right)(\xi) \leq 0, \\
& d_{2}\left[J_{2} * \underline{\phi}_{2}\right](\xi)-c \underline{\phi}_{2}^{\prime}(\xi)+r_{2} \underline{\phi}_{2}(\xi) H_{2}\left(\underline{\phi}_{1}, \bar{\phi}_{2}, \underline{\phi}_{2}\right)(\xi) \geq 0,
\end{aligned}
$$

for $\xi \in \mathbb{R} \backslash E$.

Then (2.1) has a positive solution $\left(\phi_{1}(\xi), \phi_{2}(\xi)\right)$ such that

$$
\underline{\phi}_{1}(\xi) \leq \phi_{1}(\xi) \leq \bar{\phi}_{1}(\xi), \quad \underline{\phi}_{2}(\xi) \leq \phi_{2}(\xi) \leq \bar{\phi}_{2}(\xi), \quad \xi \in \mathbb{R} .
$$

Remark 2.5 Here, $\left(\bar{\phi}_{1}(\xi), \bar{\phi}_{2}(\xi)\right),\left(\underline{\phi}_{1}(\xi), \underline{\phi}_{2}(\xi)\right)$ are a pair of generalized upper and lower solutions of (2.1). Therefore, the existence of traveling wave solutions is deduced to the existence of generalized upper and lower solutions, of which the recipe has been earlier utilized in delayed reaction-diffusion systems by Ma [25] and Wu and Zou [26] for quasimonotone systems, and by Huang and Zou [27] for predator-prey systems. When the dispersal models are involved, we also refer to [20, 21, 28-31].

\section{Existence of traveling wave solutions}

In this section, we shall present the existence of traveling wave solutions for any $c \geq c^{*}$. When the wave speed is large, there exists a positive traveling wave solution.

Theorem 3.1 If $c>c^{*}$, then (2.1) has a positive solution $\left(\phi_{1}(\xi), \phi_{2}(\xi)\right)$ such that

$$
0<\phi_{1}(\xi)<1, \quad 0<\phi_{2}(\xi)<1+c_{2}, \quad \xi \in \mathbb{R}
$$

and

$$
\lim _{\xi \rightarrow-\infty}\left(\phi_{1}(\xi), \phi_{2}(\xi)\right)=(0,0), \quad \lim _{\xi \rightarrow-\infty}\left(\phi_{1}(\xi) e^{-\lambda_{1}^{c} \xi}, \phi_{2}(\xi) e^{-\lambda_{2}^{c} \xi}\right)=(1,1)
$$


Proof We shall prove it by Lemma 2.4, and first construct generalized upper and lower solutions. For convenience, we denote $\lambda_{i}^{c}$ by $\lambda_{i}$ for simplicity, and we prove the result for any fixed $c>c^{*}$.

Define continuous functions

$$
\underline{\phi}_{1}(\xi)=\max \left\{e^{\lambda_{1} \xi}-q e^{\eta \lambda_{1} \xi}, 0\right\}, \quad \underline{\phi}_{2}(\xi)=\max \left\{e^{\lambda_{2} \xi}-q e^{\eta \lambda_{2} \xi}, 0\right\}
$$

and

$$
\bar{\phi}_{1}(\xi)=\min \left\{e^{\lambda_{1} \xi}, 1\right\}, \quad \bar{\phi}_{2}(\xi)=\min \left\{e^{\lambda_{2} \xi}+p e^{\eta \lambda_{2} \xi}, 1+c_{2}\right\}
$$

where

$$
\eta \in\left(1, \min \left\{\frac{\lambda_{3}}{\lambda_{1}}, \frac{\lambda_{4}}{\lambda_{2}}, \frac{\lambda_{1}+\lambda_{2}}{\lambda_{1}}, \frac{\lambda_{1}+\lambda_{2}}{\lambda_{2}}\right\}\right)
$$

and $p>1, q>1$ are constants, of which the definitions will be clarified later. We now show these functions satisfy (2.5)-(2.8) if they are differentiable.

If $\bar{\phi}_{1}(\xi)=1<e^{\lambda_{1} \xi}$, then $H_{1}\left(\bar{\phi}_{1}, \underline{\phi}_{1}, \underline{\phi}_{2}\right)(\xi) \leq 0$ such that $(2.5)$ is clear. Otherwise, $\bar{\phi}_{1}(\xi)=$ $e^{\lambda_{1} \xi}<1$ implies that

$$
\begin{aligned}
& d_{1}\left[J_{1} * \bar{\phi}_{1}\right](\xi)-c \bar{\phi}_{1}^{\prime}(\xi)+r_{1} \bar{\phi}_{1}(\xi) H_{1}\left(\bar{\phi}_{1}, \underline{\phi}_{1}, \underline{\phi}_{2}\right)(\xi) \\
& \quad \leq d_{1}\left[J_{1} * \bar{\phi}_{1}\right](\xi)-c \bar{\phi}_{1}^{\prime}(\xi)+r_{1} \bar{\phi}_{1}(\xi) \\
& \quad=d_{1}\left[\int_{\mathbb{R}} J_{1}(y) \bar{\phi}_{1}(\xi-y) d y-e^{\lambda_{1} \xi}\right]-c \lambda_{1} e^{\lambda_{1} \xi}+r_{1} e^{\lambda_{1} \xi} \\
& \quad \leq d_{1}\left[\int_{\mathbb{R}} J_{1}(y) e^{\lambda_{1}(\xi-y)} d y-e^{\lambda_{1} \xi}\right]-c \lambda_{1} e^{\lambda_{1} \xi}+r_{1} e^{\lambda_{1} \xi} \\
& =e^{\lambda_{1} \xi}\left\{d_{1}\left[\int_{\mathbb{R}} J_{1}(y) e^{\lambda_{1} y} d y-1\right]-c \lambda_{1}+r_{1}\right\} \\
& =0,
\end{aligned}
$$

which implies what we wanted.

If $\bar{\phi}_{2}(\xi)=1+c_{2}<e^{\lambda_{2} \xi}+p e^{\eta \lambda_{2} \xi}$, then $H_{2}\left(\bar{\phi}_{1}, \underline{\phi}_{2}, \bar{\phi}_{2}\right)(\xi) \leq 0$ such that (2.7) is clear. Otherwise, $\bar{\phi}_{2}(\xi)=e^{\lambda_{2} \xi}+p e^{\eta \lambda_{2} \xi}<1+c_{2}$ such that

$$
\begin{aligned}
& r_{2} \bar{\phi}_{2}(\xi) H_{2}\left(\bar{\phi}_{1}, \underline{\phi}_{2}, \bar{\phi}_{2}\right)(\xi) \\
& \quad=r_{2} \bar{\phi}_{2}(\xi)\left[1-\bar{\phi}_{2}(\xi)-b_{2} \int_{-\tau}^{0} \underline{\phi}_{2}(\xi+c s) d \eta_{22}(s)+c_{2} \int_{-\tau}^{0} \bar{\phi}_{1}(\xi+c s) d \eta_{21}(s)\right] \\
& \quad \leq r_{2} \bar{\phi}_{2}(\xi)\left[1+c_{2} \int_{-\tau}^{0} \bar{\phi}_{1}(\xi+c s) d \eta_{21}(s)\right] \\
& \quad \leq r_{2} \bar{\phi}_{2}(\xi)\left[1+c_{2} e^{\lambda_{1} \xi}\right] \\
& \quad=r_{2}\left[e^{\lambda_{2} \xi}+p e^{\eta \lambda_{2} \xi}\right]\left[1+c_{2} e^{\lambda_{1} \xi}\right] \\
& \quad=r_{2}\left[e^{\lambda_{2} \xi}+p e^{\eta \lambda_{2} \xi}\right]+r_{2} c_{2} e^{\lambda_{1} \xi}\left[e^{\lambda_{2} \xi}+p e^{\eta \lambda_{2} \xi}\right]
\end{aligned}
$$


and

$$
\begin{aligned}
d_{2} J_{2} & \left.* \bar{\phi}_{2}\right](\xi)-c \bar{\phi}_{2}^{\prime}(\xi)+r_{2} \bar{\phi}_{2}(\xi) H_{2}\left(\bar{\phi}_{1}, \underline{\phi}_{2}, \bar{\phi}_{2}\right)(\xi) \\
= & d_{2}\left[\int_{\mathbb{R}} J_{2}(y) \bar{\phi}_{2}(\xi-y) d y-\left(e^{\lambda_{2} \xi}+p e^{\eta \lambda_{2} \xi}\right)\right] \\
& -c\left(\lambda_{2} e^{\lambda_{2} \xi}+p \eta \lambda_{2} e^{\eta \lambda_{2} \xi}\right)+r_{2} \bar{\phi}_{2}(\xi) H_{2}\left(\bar{\phi}_{1}, \underline{\phi}_{2}, \bar{\phi}_{2}\right)(\xi) \\
\leq & d_{2}\left[\int_{\mathbb{R}} J_{2}(y)\left[e^{\lambda_{2}(\xi-y)}+p e^{\eta \lambda_{2}(\xi-y)}\right] d y-\left(e^{\lambda_{2} \xi}+p e^{\eta \lambda_{2} \xi}\right)\right] \\
& -c\left(\lambda_{2} e^{\lambda_{2} \xi}+p \eta \lambda_{2} e^{\eta \lambda_{2} \xi}\right)+r_{2} \bar{\phi}_{2}(\xi) H_{2}\left(\bar{\phi}_{1}, \underline{\phi}_{2}, \bar{\phi}_{2}\right)(\xi) \\
\leq & d_{2}\left[\int_{\mathbb{R}} J_{2}(y)\left[e^{\lambda_{2}(\xi-y)}+p e^{\eta \lambda_{2}(\xi-y)}\right] d y-\left(e^{\lambda_{2} \xi}+p e^{\eta \lambda_{2} \xi}\right)\right] \\
& -c\left(\lambda_{2} e^{\lambda_{2} \xi}+p \eta \lambda_{2} e^{\eta \lambda_{2} \xi}\right)+r_{2}\left[e^{\lambda_{2} \xi}+p e^{\eta \lambda_{2} \xi}\right]+r_{2} c_{2} e^{\lambda_{1} \xi}\left[e^{\lambda_{2} \xi}+p e^{\eta \lambda_{2} \xi}\right] \\
= & p\left\{d_{2}\left[\int_{\mathbb{R}} J_{2}(y) e^{\eta \lambda_{2}(\xi-y)} d y-e^{\eta \lambda_{2} \xi}\right]-c \eta \lambda_{2} e^{\eta \lambda_{2} \xi}+r_{2} e^{\eta \lambda_{2} \xi}\right\} \\
& +r_{2} c_{2} e^{\lambda_{1} \xi}\left[e^{\lambda_{2} \xi}+p e^{\eta \lambda_{2} \xi}\right] \\
= & p \Theta_{2}\left(\eta \lambda_{2}, c\right) e^{\eta \lambda_{2} \xi}+r_{2} c_{2} e^{\lambda_{1} \xi}\left[e^{\lambda_{2} \xi}+p e^{\eta \lambda_{2} \xi}\right] \\
= & e^{\eta \lambda_{2} \xi}\left[p \Theta_{2}\left(\eta \lambda_{2}, c\right) / 2+r_{2} c_{2} e^{\left(\lambda_{1}+\lambda_{2}-\eta \lambda_{2}\right) \xi}\right]+p e^{\eta \lambda_{2} \xi}\left[\Theta_{2}\left(\eta \lambda_{2}, c\right) / 2+r_{2} c_{2} e^{\lambda_{1} \xi}\right] .
\end{aligned}
$$

Note that

$$
\eta \lambda_{2} \xi<\ln \frac{1+c_{2}}{p}
$$

then there exists $p_{1}>1+c_{2}$ such that $p=p_{1}$ leads to

$$
p \Theta_{2}\left(\eta \lambda_{2}, c\right) / 2+r_{2} c_{2} e^{\left(\lambda_{1}+\lambda_{2}-\eta \lambda_{2}\right) \xi}<0, \quad \Theta_{2}\left(\eta \lambda_{2}, c\right) / 2+r_{2} c_{2} e^{\lambda_{1} \xi}<0
$$

since $\lambda_{1}+\lambda_{2}-\eta \lambda_{2}>0, \xi<0$ and $\Theta_{2}\left(\eta \lambda_{2}, c\right)<0$ is a constant.

When $\underline{\phi}_{1}(\xi)=0>e^{\lambda_{1} \xi}-q e^{\eta \lambda_{1} \xi}$, then $H_{1}\left(\underline{\phi}_{1}, \bar{\phi}_{1}, \bar{\phi}_{2}\right)(\xi)=0$ such that (2.6) is clear. Otherwise, $\underline{\phi}_{1}(\xi)=e^{\lambda_{1} \xi}-q e^{\eta \lambda_{1} \xi}>0$. Firstly, let $q>q_{1}>1$ such that $e^{\lambda_{1} \xi}-q_{1} e^{\eta \lambda_{1} \xi}>0$ implies $\xi<0$ and

$$
\bar{\phi}_{2}(\xi)<2 e^{\lambda_{2} \xi}
$$

which is admissible once $p$ is fixed. Therefore, the monotonicity and $q>q_{1}$ indicate

$$
\begin{aligned}
& r_{1} \underline{\phi}_{1}(\xi) H_{1}\left(\phi_{1}, \psi_{1}, \phi_{2}\right)(\xi) \\
& \quad=r_{1} \underline{\phi}_{1}(\xi)\left[1-\underline{\phi}_{1}(\xi)-b_{1} \int_{-\tau}^{0} \bar{\phi}_{1}(\xi+c s) d \eta_{11}(s)-c_{1} \int_{-\tau}^{0} \bar{\phi}_{2}(\xi+c s) d \eta_{12}(s)\right] \\
& \quad \geq r_{1} \underline{\phi}_{1}(\xi)-r_{1} \underline{\phi}_{1}^{2}(\xi)-r_{1} b_{1} \underline{\phi}_{1}(\xi) \bar{\phi}_{1}(\xi)-2 r_{1} c_{1} e^{\lambda_{2} \xi} \underline{\phi}_{1}(\xi) \\
& \quad \geq r_{1} \underline{\phi}_{1}(\xi)-r_{1}\left(1+b_{1}\right) e^{2 \lambda_{1} \xi}-2 r_{1} c_{1} e^{\left(\lambda_{1}+\lambda_{2}\right) \xi} \\
& \quad=r_{1} e^{\lambda_{1} \xi}-r_{1} q_{1} e^{\eta \lambda_{1} \xi}-r_{1}\left(1+b_{1}\right) e^{2 \lambda_{1} \xi}-2 r_{1} c_{1} e^{\left(\lambda_{1}+\lambda_{2}\right) \xi} .
\end{aligned}
$$


By what we have done, (2.6) is true once

$$
\begin{aligned}
d_{1}\left[J_{1}\right. & \left.* \underline{\phi}_{1}\right](\xi)-c \underline{\phi}_{1}^{\prime}(\xi)+r_{1} e^{\lambda_{1} \xi}-r_{1} q_{1} e^{\eta \lambda_{1} \xi}-r_{1}\left(1+b_{1}\right) e^{2 \lambda_{1} \xi}-2 r_{1} c_{1} e^{\left(\lambda_{1}+\lambda_{2}\right) \xi} \\
\geq & d_{1}\left[\int_{\mathbb{R}} J_{1}(y)\left(e^{\lambda_{1}(\xi-y)}-q e^{\eta \lambda_{1}(\xi-y)}\right) d y-\left(e^{\lambda_{1} \xi}-q e^{\eta \lambda_{1} \xi}\right)\right] \\
& -\left(c \lambda_{1} e^{\lambda_{1} \xi}-c q \eta \lambda_{1} e^{\eta \lambda_{1} \xi}\right)+r_{1} e^{\lambda_{1} \xi}-r_{1} q e^{\eta \lambda_{1} \xi} \\
& -r_{1}\left(1+b_{1}\right) e^{2 \lambda_{1} \xi}-2 r_{1} c_{1} e^{\left(\lambda_{1}+\lambda_{2}\right) \xi} \\
=- & q e^{\eta \lambda_{1} \xi}\left\{d_{1}\left[\int_{\mathbb{R}} J_{1}(y) e^{\eta \lambda_{1} y} d y-1\right]-c \eta \lambda_{1}+r_{1}\right\} \\
& -r_{1}\left(1+b_{1}\right) e^{2 \lambda_{1} \xi}-2 r_{1} c_{1} e^{\left(\lambda_{1}+\lambda_{2}\right) \xi} \\
= & -q \Theta_{1}\left(\eta \lambda_{1}, c\right) e^{\eta \lambda_{1} \xi}-r_{1}\left(1+b_{1}\right) e^{2 \lambda_{1} \xi}-2 r_{1} c_{1} e^{\left(\lambda_{1}+\lambda_{2}\right) \xi} \\
\geq & 0 .
\end{aligned}
$$

Let

$$
q>-\frac{r_{1}\left(1+b_{1}\right)+2 r_{1} c_{1}}{\Theta_{1}\left(\eta \lambda_{1}, c\right)}+q_{1}:=q_{2},
$$

then (3.2) holds since $\xi<0$ and

$$
e^{\eta \lambda_{1} \xi}>e^{2 \lambda_{1} \xi}>0, \quad e^{\eta \lambda_{1} \xi}>e^{\left(\lambda_{1}+\lambda_{2}\right) \xi}>0 .
$$

The verification of (2.6) is finished.

We now consider (2.8), which is clear if $\underline{\phi}_{2}(\xi)=0>e^{\lambda_{2} \xi}-q e^{\eta \lambda_{2} \xi}$. If $\underline{\phi}_{2}(\xi)=e^{\lambda_{2} \xi}-q e^{\eta \lambda_{2} \xi}>$ 0 , we first select $q_{3} \geq q_{2}$ implies

$$
\bar{\phi}_{2}(\xi)<2 e^{\lambda_{2} \xi}
$$

for any $q \geq q_{3}$, which is admissible for fixed $p=p_{1}$. Then

$$
\begin{aligned}
& r_{2} \underline{\phi}_{2}(\xi) H_{2}\left(\underline{\phi}_{1}, \bar{\phi}_{2}, \underline{\phi}_{2}\right)(\xi) \\
& \quad=r_{2} \underline{\phi}_{2}(\xi)\left[1-\underline{\phi}_{2}(\xi)-b_{2} \int_{-\tau}^{0} \bar{\phi}_{2}(\xi+c s) d \eta_{22}(s)+c_{2} \int_{-\tau}^{0} \underline{\phi}_{1}(\xi+c s) d \eta_{21}(s)\right] \\
& \quad \geq r_{2} \underline{\phi}_{2}(\xi)\left[1-\underline{\phi}_{2}(\xi)-b_{2} \int_{-\tau}^{0} \bar{\phi}_{2}(\xi+c s) d \eta_{22}(s)\right] \\
& \geq r_{2} \underline{\phi}_{2}(\xi)\left[1-e^{\lambda_{2} \xi}-2 b_{2} e^{\lambda_{2} \xi}\right] \\
& \quad=r_{2} \underline{\phi}_{2}(\xi)-r_{2}\left(1+2 b_{2}\right) \underline{\phi}_{2}(\xi) e^{\lambda_{2} \xi} \\
& \geq r_{2}\left(e^{\lambda_{2} \xi}-q e^{\eta \lambda_{2} \xi}\right)-r_{2}\left(1+2 b_{2}\right) e^{2 \lambda_{2} \xi} .
\end{aligned}
$$

Therefore, if

$$
q>q_{3}-\frac{r_{2}\left(1+2 b_{2}\right)}{\Theta_{2}\left(\eta \lambda_{2}, c\right)}:=q_{4}
$$


then (2.8) holds since

$$
\begin{aligned}
d_{2} & \left.J_{2} * \underline{\phi}_{2}\right](\xi)-c \underline{\phi}_{2}^{\prime}(\xi)+r_{2} \underline{\phi}_{2}(\xi) H_{2}\left(\underline{\phi}_{1}, \bar{\phi}_{2}, \underline{\phi}_{2}\right)(\xi) \\
\geq & d_{2}\left[\int_{\mathbb{R}} J_{2}(y)\left[e^{\lambda_{2}(\xi-y)}-q e^{\eta \lambda_{2}(\xi-y)}\right] d y-\left(e^{\lambda_{2} \xi}-q e^{\eta \lambda_{2} \xi}\right)\right] \\
& -c\left(\lambda_{2} e^{\lambda_{2} \xi}-q \eta \lambda_{2} e^{\eta \lambda_{2} \xi}\right)+r_{2}\left(e^{\lambda_{2} \xi}-q e^{\eta \lambda_{2} \xi}\right)-r_{2}\left(1+2 b_{2}\right) e^{2 \lambda_{2} \xi} \\
= & -q e^{\eta \lambda_{2} \xi}\left\{d_{2}\left[\int_{\mathbb{R}} J_{2}(y) e^{\eta \lambda_{2} y} d y-1\right]-c \eta \lambda_{2}+r_{2}\right\}-r_{2}\left(1+2 b_{2}\right) e^{2 \lambda_{2} \xi} \\
= & -q \Theta_{2}\left(\eta \lambda_{2}, c\right) e^{\eta \lambda_{2} \xi}-r_{2}\left(1+2 b_{2}\right) e^{2 \lambda_{2} \xi} \\
\geq & 0, \quad \xi<0 .
\end{aligned}
$$

Summarizing what we have done, it suffices to verify that (3.1) is true. We now show $\phi_{1}(\xi)>0, \xi \in \mathbb{R}$. If $\phi_{1}\left(\xi_{0}\right)=0$, then it arrives the minimal and so $\phi_{1}^{\prime}\left(\xi_{0}\right)=0$, which further implies that

$$
\int_{\mathbb{R}} J_{1}(y) \phi_{1}\left(\xi_{0}-y\right) d y=0 .
$$

Therefore, $\phi_{1}(\xi)=0$ on an interval. Repeating the process, we see that $\phi_{1}(\xi)=0, \xi \in \mathbb{R}$. A contradiction occurs since $\underline{\phi}_{1}(\xi)>0$ if $-\xi$ is large. Similarly, we can verify (3.1). The proof is complete.

Theorem 3.2 Assume that $c^{*}=c_{1}^{*}>c_{2}^{*}$. Further suppose that $k_{1}(y)$ admits compact support. Then (2.1) with $c=c^{*}$ has a positive solution $\left(\phi_{1}(\xi), \phi_{2}(\xi)\right)$ such that

$$
0<\phi_{1}(\xi)<1,0<\phi_{2}(\xi)<1+c_{2}, \xi \in \mathbb{R}, \quad \lim _{\xi \rightarrow-\infty}\left(\phi_{1}(\xi), \phi_{2}(\xi)\right)=(0,0)
$$

and

$$
\phi_{1}(\xi) \sim \mathcal{O}\left(-\xi e^{\lambda_{1}^{*} \xi}\right), \quad \phi_{2}(\xi) \sim \mathcal{O}\left(e^{\lambda_{2} \xi}\right), \quad \xi \rightarrow-\infty
$$

Proof By Lemma 2.3, $\Theta_{1}\left(\lambda, c^{*}\right)$ arrives at its minimum when $\lambda=\lambda_{1}^{*}$, and so

$$
d_{1} \int_{\mathbb{R}} J_{1}(y) y e^{\lambda_{1}^{*} y} d y=c^{*}
$$

Let $S>0$ be a constant such that $k_{1}(y)=0,|y|>S$. Moreover, let $\eta>1$ such that

$$
\lambda_{1}^{*} / 2+\lambda_{2}-\eta \lambda_{2}>0, \quad \Theta_{2}\left(\eta \lambda_{2}, c^{*}\right)<0
$$

Consider the continuous function $-L \xi e^{\lambda_{1}^{*} \xi}, \xi<0$, where $L>0$ is a constant. Clearly, if $L>1$ is large, then

$$
\max _{\xi<0}\left\{-L \xi e^{\lambda_{1}^{*} \xi}\right\}>1, \quad \xi_{2}-\xi_{1}>2 S+c^{*} \tau,
$$


where $\xi_{2}, \xi_{1}$ with $\xi_{2}-\xi_{1}>0$ are two roots of $-L \xi e^{\lambda_{1}^{*} \xi}=1$. Moreover, let $q>L$ be a constant clarified later, then there exists $\xi_{3}=-q^{2} / L^{2}<-1$ such that

$$
(-L \xi-q \sqrt{-\xi}) e^{\lambda_{1}^{*} \xi}>0, \quad \xi<\xi_{3} .
$$

By the above constants, define the continuous functions

$$
\begin{aligned}
& \underline{\phi}_{1}(\xi)= \begin{cases}(-L \xi-q \sqrt{-\xi}) e^{\lambda_{1}^{*} \xi}, & \xi<\xi_{3}, \\
0, & \xi \geq \xi_{3},\end{cases} \\
& \bar{\phi}_{1}(\xi)= \begin{cases}-L \xi e^{\lambda_{1}^{*} \xi}, & \xi<\xi_{1}, \\
1, & \xi \geq \xi_{1},\end{cases}
\end{aligned}
$$

and

$$
\underline{\phi}_{2}(\xi)=\max \left\{e^{\lambda_{2} \xi}-q e^{\eta \lambda_{2} \xi}, 0\right\}, \quad \bar{\phi}_{2}(\xi)=\min \left\{e^{\lambda_{2} \xi}+p e^{\eta \lambda_{2} \xi}, 1+c_{2}\right\},
$$

where $p>1, q>1$ are constants, of which the definition will be further illustrated later. We now show these functions satisfy (2.5)-(2.8) if they are differentiable.

If $\bar{\phi}_{1}(\xi)=1$, then $H_{1}\left(\bar{\phi}_{1}, \underline{\phi}_{1}, \underline{\phi}_{2}\right)(\xi) \leq 0$ such that $(2.5)$ is clear. Otherwise, $\bar{\phi}_{1}(\xi)=$ $-L \xi e^{\lambda_{1}^{*} \xi}<1$ implies that

$$
r_{1} \bar{\phi}_{1}(\xi) H_{1}\left(\bar{\phi}_{1}, \underline{\phi}_{1}, \underline{\phi}_{2}\right)(\xi) \leq r_{1} \bar{\phi}_{1}(\xi)=-r_{1} L \xi e^{\lambda_{1}^{*} \xi}, \quad \xi<\xi_{1},
$$

and (3.3) indicates that

$$
\begin{aligned}
d_{1}\left[J_{1}\right. & \left.* \bar{\phi}_{1}\right](\xi)-c^{*} \bar{\phi}_{1}^{\prime}(\xi)+r_{1} \bar{\phi}_{1}(\xi) H_{1}\left(\bar{\phi}_{1}, \underline{\phi}_{1}, \underline{\phi}_{2}\right)(\xi) \\
\leq & d_{1}\left[J_{1} * \bar{\phi}_{1}\right](\xi)-c^{*} \bar{\phi}_{1}^{\prime}(\xi)+r_{1} \bar{\phi}_{1}(\xi) \\
\leq & -d_{1} L\left[\int_{\mathbb{R}} J_{1}(y)(\xi-y) e^{\lambda_{1}^{*}(\xi-y)} d y-\xi e^{\lambda_{1}^{*} \xi}\right] \\
& +c^{*} L e^{\lambda_{1}^{*} \xi}+c^{*} \lambda_{1}^{*} L \xi e^{\lambda_{1}^{*} \xi}-r_{1} L \xi e^{\lambda_{1}^{*} \xi} \\
= & -d_{1} L\left[\xi \int_{\mathbb{R}} J_{1}(y) e^{\lambda_{1}^{*}(\xi-y)} d y-\xi e^{\lambda_{1}^{*} \xi}-\int_{\mathbb{R}} J_{1}(y) y e^{\lambda_{1}^{*}(\xi-y)} d y\right] \\
& +c^{*} L e^{\lambda_{1}^{*} \xi}+c^{*} \lambda_{1}^{*} L \xi e^{\lambda_{1}^{*} \xi}-r_{1} L \xi e^{\lambda_{1}^{*} \xi} \\
= & -L \xi e^{\lambda_{1}^{*} \xi}\left\{d_{1}\left[\int_{\mathbb{R}} J_{1}(y) e^{\lambda_{1}^{*} y} d y-1\right]-c^{*} \lambda_{1}^{*}+r_{1}\right\} \\
& +d_{1} L e^{\lambda_{1}^{*} \xi}\left[\int_{\mathbb{R}} J_{1}(y) y e^{-\lambda_{1}^{*} y} d y\right]+c^{*} L e^{\lambda_{1}^{*} \xi} \\
= & 0,
\end{aligned}
$$

which implies what we wanted.

If $\bar{\phi}_{2}(\xi)=1+c_{2}<e^{\lambda_{2} \xi}+p e^{\eta \lambda_{2} \xi}$, then $H_{2}\left(\bar{\phi}_{1}, \underline{\phi}_{2}, \bar{\phi}_{2}\right)(\xi) \leq 0$ such that (2.7) is clear. Otherwise, let $p_{2}>0$ such that $\bar{\phi}_{2}(\xi)=e^{\lambda_{2} \xi}+p e^{\eta \lambda_{2} \xi}<1+c_{2}$ with $p \geq p_{2}$ implies that

$$
\bar{\phi}_{1}(\xi)<e^{\lambda_{1}^{*} \xi / 2},
$$


which is evident by simple limit analysis. Thus, the monotonicity implies

$$
\begin{aligned}
& r_{2} \bar{\phi}_{2}(\xi) H_{2}\left(\bar{\phi}_{1}, \underline{\phi}_{2}, \bar{\phi}_{2}\right)(\xi) \\
& \quad=r_{2} \bar{\phi}_{2}(\xi)\left[1-\bar{\phi}_{2}(\xi)-b_{2} \int_{-\tau}^{0} \underline{\phi}_{2}\left(\xi+c^{*} s\right) d \eta_{22}(s)+c_{2} \int_{-\tau}^{0} \bar{\phi}_{1}\left(\xi+c^{*} s\right) d \eta_{21}(s)\right] \\
& \quad \leq r_{2} \bar{\phi}_{2}(\xi)\left[1+c_{2} \int_{-\tau}^{0} \bar{\phi}_{1}\left(\xi+c^{*} s\right) d \eta_{21}(s)\right] \\
& \quad \leq r_{2} \bar{\phi}_{2}(\xi)\left[1+c_{2} e^{\lambda_{1}^{*} \xi / 2}\right] \\
& \quad=r_{2}\left[e^{\lambda_{2} \xi}+p e^{\eta \lambda_{2} \xi}\right]\left[1+c_{2} e^{\lambda_{1}^{*} \xi / 2}\right] \\
& \quad=r_{2}\left[e^{\lambda_{2} \xi}+p e^{\eta \lambda_{2} \xi}\right]+r_{2} c_{2} e^{\lambda_{1}^{*} \xi / 2}\left[e^{\lambda_{2} \xi}+p e^{\eta \lambda_{2} \xi}\right]
\end{aligned}
$$

and

$$
\begin{aligned}
d_{2}\left[J_{2}\right. & \left.* \bar{\phi}_{2}\right](\xi)-c^{*} \bar{\phi}_{2}^{\prime}(\xi)+r_{2} \bar{\phi}_{2}(\xi) H_{2}\left(\bar{\phi}_{1}, \underline{\phi}_{2}, \bar{\phi}_{2}\right)(\xi) \\
= & d_{2}\left[\int_{\mathbb{R}} J_{2}(y) \bar{\phi}_{2}(\xi-y) d y-\left(e^{\lambda_{2} \xi}+p e^{\eta \lambda_{2} \xi}\right)\right] \\
& -c^{*}\left(\lambda_{2} e^{\lambda_{2} \xi}+p \eta \lambda_{2} e^{\eta \lambda_{2} \xi}\right)+r_{2} \bar{\phi}_{2}(\xi) H_{2}\left(\bar{\phi}_{1}, \underline{\phi}_{2}, \bar{\phi}_{2}\right)(\xi) \\
\leq & d_{2}\left[\int_{\mathbb{R}} J_{2}(y)\left[e^{\lambda_{2}(\xi-y)}+p e^{\eta \lambda_{2}(\xi-y)}\right] d y-\left(e^{\lambda_{2} \xi}+p e^{\eta \lambda_{2} \xi}\right)\right] \\
& -c^{*}\left(\lambda_{2} e^{\lambda_{2} \xi}+p \eta \lambda_{2} e^{\eta \lambda_{2} \xi}\right)+r_{2} \bar{\phi}_{2}(\xi) H_{2}\left(\bar{\phi}_{1}, \underline{\phi}_{2}, \bar{\phi}_{2}\right)(\xi) \\
\leq & d_{2}\left[\int_{\mathbb{R}} J_{2}(y)\left[e^{\lambda_{2}(\xi-y)}+p e^{\eta \lambda_{2}(\xi-y)}\right] d y-\left(e^{\lambda_{2} \xi}+p e^{\eta \lambda_{2} \xi}\right)\right] \\
& -c^{*}\left(\lambda_{2} e^{\lambda_{2} \xi}+p \eta \lambda_{2} e^{\eta \lambda_{2} \xi}\right)+r_{2}\left[e^{\lambda_{2} \xi}+p e^{\eta \lambda_{2} \xi}\right]+r_{2} c_{2}^{*} e^{\lambda_{1}^{*} \xi / 2}\left[e^{\lambda_{2} \xi}+p e^{\eta \lambda_{2} \xi}\right] \\
= & p e^{\eta \lambda_{2} \xi}\left\{d_{2}\left[\int_{\mathbb{R}} J_{2}(y) e^{\eta \lambda_{2} y} d y-1\right]-c^{*} \eta \lambda_{2}+r_{2}\right\}+r_{2} c_{2} e^{\lambda_{1}^{*} \xi / 2}\left[e^{\lambda_{2} \xi}+p e^{\eta \lambda_{2} \xi}\right] \\
= & p \Theta_{2}\left(\eta \lambda_{2}, c^{*}\right) e^{\eta \lambda_{2} \xi}+r_{2} c_{2} e^{\lambda_{1}^{*} \xi / 2}\left[e^{\lambda_{2} \xi}+p e^{\eta \lambda_{2} \xi}\right] \\
= & e^{\eta \lambda_{2} \xi}\left[p \Theta_{2}\left(\eta \lambda_{2}, c^{*}\right) / 2+r_{2} c_{2} e^{\left(\lambda_{1}^{*} / 2+\lambda_{2}-\eta \lambda_{2}\right) \xi}\right] \\
& +p e^{\eta \lambda_{2} \xi}\left[\Theta_{2}\left(\eta \lambda_{2}, c^{*}\right) / 2+r_{2} c_{2} e^{\lambda_{1}^{*} \xi / 2}\right] .
\end{aligned}
$$

Note that

$$
\eta \lambda_{2} \xi<\ln \frac{1+c_{2}}{p}
$$

then there exists $p_{3}>p_{2}+1+c_{2}$ such that $p \geq p_{3}$ leads to

$$
\begin{aligned}
& p \Theta_{2}\left(\eta \lambda_{2}, c^{*}\right) / 2+r_{2} c_{2} e^{\left(\lambda_{1} / 2+\lambda_{2}-\eta \lambda_{2}\right) \xi}<0, \\
& \Theta_{2}\left(\eta \lambda_{2}, c^{*}\right) / 2+r_{2} c_{2} e^{\lambda_{1}^{*} \xi / 2}<0
\end{aligned}
$$

since $\lambda_{1}^{*} / 2+\lambda_{2}-\eta \lambda_{2}>0, \xi<0$ and $\Theta_{2}\left(\eta \lambda_{2}, c^{*}\right)<0$ is a constant. Now, we fix it by $p=p_{3}$. 
When $\underline{\phi}_{1}(\xi)=0$ with $\xi<\xi_{3}$, then $H_{1}\left(\underline{\phi}_{1}, \bar{\phi}_{1}, \bar{\phi}_{2}\right)(\xi)=0$ such that (2.6) is clear. Otherwise, if $\xi \geq \xi_{3}$, then $\underline{\phi}_{1}(\xi)=(-L \xi-q \sqrt{-\xi}) e^{\lambda_{1}^{*} \xi}>0$. Firstly, let $q>q_{1}>1$ such that $-L \xi-q \sqrt{-\xi}>0$ implies $\xi<0$ and

$$
\bar{\phi}_{2}(\xi)<2 e^{\lambda_{2} \xi}, \quad \underline{\phi}_{1}(\xi) \leq \bar{\phi}_{1}(\xi)<e^{\theta \lambda_{1}^{*} \xi}
$$

for some $\theta \in\left[\frac{2}{3}, 1\right)$ with $\theta \lambda_{1}^{*}+\lambda_{2}>\lambda_{1}^{*}$, which is admissible once $p$ is fixed. Therefore, $q>q_{1}$ indicates

$$
\begin{aligned}
& r_{1} \underline{\phi}_{1}(\xi) H_{1}\left(\underline{\phi}_{1}, \bar{\phi}_{1}, \bar{\phi}_{2}\right)(\xi) \\
& \quad=r_{1} \underline{\phi}_{1}(\xi)\left[1-\underline{\phi}_{1}(\xi)-b_{1} \int_{-\tau}^{0} \bar{\phi}_{1}\left(\xi+c^{*} s\right) d \eta_{11}(s)-c_{1} \int_{-\tau}^{0} \bar{\phi}_{2}\left(\xi+c^{*} s\right) d \eta_{12}(s)\right] \\
& \quad \geq r_{1} \underline{\phi}_{1}(\xi)-r_{1} \underline{\phi}_{1}^{2}(\xi)-r_{1} b_{1} \underline{\phi}_{1}(\xi) \bar{\phi}_{1}(\xi)-2 r_{1} c_{1} e^{\lambda_{2} \xi} \underline{\phi}_{1}(\xi) \\
& \quad \geq r_{1} \underline{\phi}_{1}(\xi)-r_{1}\left(1+b_{1}\right) e^{2 \theta \lambda_{1}^{*} \xi}-2 r_{1} c_{1} e^{\left(\theta \lambda_{1}^{*}+\lambda_{2}\right) \xi} \\
& \quad=r_{1}(-L \xi-q \sqrt{-\xi}) e^{\lambda_{1}^{*} \xi}-r_{1}\left(1+b_{1}\right) e^{2 \theta \lambda_{1}^{*} \xi}-2 r_{1} c_{1} e^{\left(\theta \lambda_{1}^{*}+\lambda_{2}\right) \xi} .
\end{aligned}
$$

Moreover, (3.3) leads to

$$
\begin{aligned}
\left.d_{1} J_{1} * \underline{\phi}_{1}\right](\xi)-c^{*} \underline{\phi}_{1}^{\prime}(\xi) & \\
= & d_{1}\left[\int_{\mathbb{R}} J_{1}(y) \underline{\phi}_{1}(\xi-y) d y-\underline{\phi}_{1}(\xi)\right]-c^{*} \underline{\phi}_{1}^{\prime}(\xi) \\
\geq & d_{1}\left\{\int_{\mathbb{R}} J_{1}(y)\left[(-L(\xi-y)-q \sqrt{-(\xi-y)}) e^{\lambda_{1}^{*}(\xi-y)}\right] d y\right. \\
& \left.-(-L \xi-q \sqrt{-\xi}) e^{\lambda_{1}^{*} \xi}\right\}-c^{*}\left[(-L \xi-q \sqrt{-\xi}) e^{\lambda_{1}^{*} \xi}\right]^{\prime} \\
= & d_{1} e^{\lambda_{1}^{*} \xi}\left[\int_{\mathbb{R}} J_{1}(y)\left[(-L(\xi-y)) e^{-\lambda_{1}^{*} y}\right] d y+L \xi\right] \\
& -q d_{1} e^{\lambda_{1}^{*} \xi}\left[\int_{\mathbb{R}} J_{1}(y) \sqrt{-(\xi-y)} e^{-\lambda_{1}^{*} y} d y-\sqrt{-\xi}\right] \\
& +c^{*} L\left(1+\lambda_{1}^{*} \xi\right) e^{\lambda_{1}^{*} \xi}+c^{*} q\left(\lambda_{1}^{*} \sqrt{-\xi}-\frac{1}{2 \sqrt{-\xi}}\right) e^{\lambda_{1}^{*} \xi} .
\end{aligned}
$$

By what we have done, (2.6) is true if

$$
\begin{aligned}
& \left.d_{1} J_{1} * \underline{\phi}_{1}\right](\xi)-c^{*} \underline{\phi}_{1}^{\prime}(\xi)+r_{1} \underline{\phi}_{1}(\xi) H_{1}\left(\underline{\phi}_{1}, \bar{\phi}_{1}, \bar{\phi}_{2}\right)(\xi) \\
& \geq d_{1} e^{\lambda_{1}^{*} \xi}\left[\int_{\mathbb{R}} J_{1}(y)\left[(-L(\xi-y)) e^{-\lambda_{1}^{*} y}\right] d y+L \xi\right] \\
& \quad-q d_{1} e^{\lambda_{1}^{*} \xi}\left[\int_{\mathbb{R}} J_{1}(y) \sqrt{-(\xi-y)} e^{-\lambda_{1}^{*} y} d y-\sqrt{-\xi}\right] \\
& \quad+c^{*} L\left(1+\lambda_{1}^{*} \xi\right) e^{\lambda_{1}^{*} \xi}+c^{*} q\left(\lambda_{1}^{*} \sqrt{-\xi}-\frac{1}{2 \sqrt{-\xi}}\right) e^{\lambda_{1}^{*} \xi} \\
& \quad+r_{1}(-L \xi-q \sqrt{-\xi}) e^{\lambda_{1}^{*} \xi}-r_{1}\left(1+b_{1}\right) e^{2 \theta \lambda_{1}^{*} \xi}-2 r_{1} c_{1} e^{\left(\theta \lambda_{1}^{*}+\lambda_{2}\right) \xi}
\end{aligned}
$$


Li et al. Boundary Value Problems （2018） 2018:49

Page 13 of 26

$$
\begin{aligned}
= & -L \xi e^{\lambda_{1}^{*} \xi}\left\{d_{1}\left[\int_{\mathbb{R}} J_{1}(y) e^{-\lambda_{1}^{*} y} d y-1\right]-c^{*} \lambda_{1}^{*}+r_{1}\right\} \\
& +d_{1} L e^{\lambda_{1}^{*} \xi}\left[\int_{\mathbb{R}} J_{1}(y) y e^{-\lambda_{1}^{*} y} d y+c^{*}\right]-q d_{1} e^{\lambda_{1}^{*} \xi}\left[\int_{\mathbb{R}} J_{1}(y) \sqrt{-(\xi-y)} e^{-\lambda_{1}^{*} y} d y-\sqrt{-\xi}\right] \\
& +c^{*} q\left(\lambda_{1}^{*} \sqrt{-\xi}-\frac{1}{2 \sqrt{-\xi}}\right) e^{\lambda_{1}^{*} \xi}-r_{1} q \sqrt{-\xi} e^{\lambda_{1}^{*} \xi}-r_{1}\left(1+b_{1}\right) e^{2 \theta \lambda_{1}^{*} \xi}-2 r_{1} c_{1} e^{\left(\theta \lambda_{1}^{*}+\lambda_{2}\right) \xi} \\
= & -q d_{1} e^{\lambda_{1}^{*} \xi}\left[\int_{\mathbb{R}} J_{1}(y) \sqrt{-(\xi-y)} e^{-\lambda_{1}^{*} y} d y-\sqrt{-\xi}\right]+c^{*} q\left(\lambda_{1}^{*} \sqrt{-\xi}-\frac{1}{2 \sqrt{-\xi}}\right) e^{\lambda_{1}^{*} \xi} \\
& -r_{1} q \sqrt{-\xi} e^{\lambda_{1}^{*} \xi}-r_{1}\left(1+b_{1}\right) e^{2 \theta \lambda_{1}^{*} \xi}-2 r_{1} c_{1} e^{\left(\theta \lambda_{1}^{*}+\lambda_{2}\right) \xi} \\
= & e^{\lambda_{1}^{*} \xi}\left\{-q d_{1}\left[\int_{\mathbb{R}} J_{1}(y) \sqrt{-(\xi-y)} e^{-\lambda_{1}^{*} y} d y-\sqrt{-\xi}\right]+c^{*} q\left(\lambda_{1}^{*} \sqrt{-\xi}-\frac{1}{2 \sqrt{-\xi}}\right)\right. \\
& \left.-r_{1} q \sqrt{-\xi}-r_{1}\left(1+b_{1}\right) e^{(2 \theta-1) \lambda_{1}^{*} \xi}-2 r_{1} c_{1} e^{\left(\theta \lambda_{1}^{*}+\lambda_{2}-\lambda_{1}^{*}\right) \xi}\right\} \\
\geq & 0
\end{aligned}
$$

or

$$
\begin{aligned}
& q\left\{-d_{1}\left[\int_{\mathbb{R}} J_{1}(y) \sqrt{-(\xi-y)} e^{-\lambda_{1}^{* y}} d y-\sqrt{-\xi}\right]+c^{*}\left(\lambda_{1}^{*} \sqrt{-\xi}-\frac{1}{2 \sqrt{-\xi}}\right)-r_{1} \sqrt{-\xi}\right\} \\
& \quad \geq r_{1}\left(1+b_{1}\right) e^{(2 \theta-1) \lambda_{1}^{*} \xi}+2 r_{1} c_{1} e^{\left(\theta \lambda_{1}^{*}+\lambda_{2}-\lambda_{1}^{*}\right) \xi} .
\end{aligned}
$$

We first analyze the left of the above inequality

$$
\begin{aligned}
-d_{1} & {\left[\int_{\mathbb{R}} J_{1}(y) \sqrt{-(\xi-y)} e^{-\lambda_{1}^{*} y} d y-\sqrt{-\xi}\right]+c^{*}\left(\lambda_{1}^{*} \sqrt{-\xi}-\frac{1}{2 \sqrt{-\xi}}\right)-r_{1} \sqrt{-\xi} } \\
= & -d_{1}\left\{\int_{\mathbb{R}} J_{1}(y)[\sqrt{-\xi}+\sqrt{-(\xi-y)}-\sqrt{-\xi}] e^{-\lambda_{1}^{*} y} d y-\sqrt{-\xi}\right\} \\
& +c^{*}\left(\lambda_{1}^{*} \sqrt{-\xi}-\frac{1}{2 \sqrt{-\xi}}\right)-r_{1} \sqrt{-\xi} \\
= & -d_{1}\left\{\int_{\mathbb{R}} J_{1}(y)[\sqrt{-(\xi-y)}-\sqrt{-\xi}] e^{-\lambda_{1}^{*} y} d y\right\}-c^{*} \frac{1}{2 \sqrt{-\xi}} \\
= & d_{1}\left\{\int_{\mathbb{R}} J_{1}(y)[\sqrt{-\xi}-\sqrt{-(\xi-y)}] e^{-\lambda_{1}^{*} y} d y\right\}-\frac{d_{1}}{2 \sqrt{-\xi}} \int_{\mathbb{R}} J_{1}(y) y e^{\lambda_{1}^{*} y} d y \\
= & d_{1} \int_{\mathbb{R}} J_{1}(y)\left[\frac{y}{2 \sqrt{-\xi}}+\sqrt{-\xi}-\sqrt{-(\xi-y)}\right] e^{-\lambda_{1}^{*} y} d y \\
= & d_{1} \int_{\mathbb{R}} J_{1}(y)\left[\frac{y}{2 \sqrt{-\xi}}-\frac{y}{\sqrt{-\xi}+\sqrt{-(\xi-y)}}\right] e^{-\lambda_{1}^{*} y} d y \\
= & d_{1} \int_{\mathbb{R}} J_{1}(y)\left[\frac{y}{2 \sqrt{-\xi}}-\frac{y}{\sqrt{-\xi}+\sqrt{-(\xi-y)}}\right] e^{-\lambda_{1}^{*} y} d y \\
= & d_{1} \int_{\mathbb{R}} J_{1}(y)\left[\frac{y[\sqrt{-(\xi-y)}-\sqrt{-\xi}]}{2 \sqrt{-\xi}[\sqrt{-\xi}+\sqrt{-(\xi-y)}]}\right] e^{-\lambda_{1}^{*} y} d y \\
= & d_{1} \int_{\mathbb{R}} J_{1}(y)\left[\frac{y^{2}}{2 \sqrt{-\xi}[\sqrt{-\xi}+\sqrt{-(\xi-y)}]^{2}}\right] e^{-\lambda_{1}^{*} y} d y
\end{aligned}
$$




$$
\begin{aligned}
& \geq d_{1} \int_{\mathbb{R}} J_{1}(y)\left[\frac{y^{2}}{2 \sqrt{-(\xi-S)}[\sqrt{-(\xi-S)}+\sqrt{-(\xi-S)}]^{2}}\right] e^{-\lambda_{1}^{*} y} d y \\
& =\frac{d_{1}}{8[-(\xi-S)]^{3 / 2}} \int_{\mathbb{R}} J_{1}(y) y^{2} e^{-\lambda_{1}^{*} y} d y .
\end{aligned}
$$

Let

$$
q \geq \frac{\max _{\xi<0}\left\{8[-(\xi-S)]^{3 / 2}\left[r_{1}\left(1+b_{1}\right) e^{(2 \theta-1) \lambda_{1}^{*} \xi}+2 r_{1} c_{1} e^{\left(\theta \lambda_{1}+\lambda_{2}-\lambda_{1}^{*}\right) \xi}\right]\right\}}{d_{1} \int_{\mathbb{R}} J_{1}(y) y^{2} e^{-\lambda_{1}^{*} y} d y}+q_{1}:=q_{2},
$$

then (3.2) holds since $\xi<0$ and

$$
(2 \theta-1) \lambda_{1}^{*}>0, \quad \theta \lambda_{1}^{*}+\lambda_{2}-\lambda_{1}^{*}>0 .
$$

The verification of (2.7) is finished.

We now consider (2.8), which is clear if $\underline{\phi}_{2}(\xi)=0>e^{\lambda_{2} \xi}-q e^{\eta \lambda_{2} \xi}$. If $\underline{\phi}_{2}(\xi)=e^{\lambda_{2} \xi}-q e^{\eta \lambda_{2} \xi}>$ 0 , we first select $q_{3} \geq q_{2}$ such that $\underline{\phi}_{2}(\xi)>0$ implies

$$
\bar{\phi}_{2}(\xi)<2 e^{\lambda_{2} \xi}
$$

for any $q \geq q_{3}$, which is admissible for fixed $p=p_{1}$. Then

$$
\begin{aligned}
& r_{2} \underline{\phi}_{2}(\xi) H_{2}\left(\underline{\phi}_{1}, \bar{\phi}_{2}, \underline{\phi}_{2}\right)(\xi) \\
& \quad=r_{2} \underline{\phi}_{2}(\xi)\left[1-\underline{\phi}_{2}(\xi)-b_{2} \int_{-\tau}^{0} \bar{\phi}_{2}\left(\xi+c^{*} s\right) d \eta_{22}(s)+c_{2} \int_{-\tau}^{0} \underline{\phi}_{1}\left(\xi+c^{*} s\right) d \eta_{21}(s)\right] \\
& \quad \geq r_{2} \underline{\phi}_{2}(\xi)\left[1-\underline{\phi}_{2}(\xi)-b_{2} \int_{-\tau}^{0} \bar{\phi}_{2}\left(\xi+c^{*} s\right) d \eta_{22}(s)\right] \\
& \quad \geq r_{2} \underline{\phi}_{2}(\xi)\left[1-e^{\lambda_{2} \xi}-2 b_{2} e^{\lambda_{2} \xi}\right] \\
& \quad=r_{2} \underline{\phi}_{2}(\xi)-r_{2} \underline{\phi}_{2}(\xi)\left[e^{\lambda_{2} \xi}+2 b_{2} e^{\lambda_{2} \xi}\right] \\
& \quad \geq r_{2}\left(e^{\lambda_{2} \xi}-q e^{\eta \lambda_{2} \xi}\right)-r_{2} e^{\lambda_{2} \xi}\left[e^{\lambda_{2} \xi}+2 b_{2} e^{\lambda_{2} \xi}\right] .
\end{aligned}
$$

Therefore, if

$$
q>q_{3}-\frac{r_{2}\left(1+2 b_{2}\right)}{\Theta_{2}\left(\eta \lambda_{2}, c^{*}\right)}:=q_{4},
$$

then (2.8) holds since

$$
\begin{aligned}
d_{2} & \left.J_{2} * \underline{\phi}_{2}\right](\xi)-c^{*} \underline{\phi}_{2}^{\prime}(\xi)+r_{2} \underline{\phi}_{2}(\xi) H_{2}\left(\underline{\phi}_{1}, \bar{\phi}_{2}, \underline{\phi}_{2}\right)(\xi) \\
\geq & d_{2}\left[\int_{\mathbb{R}} J_{2}(y)\left[e^{\lambda_{2}(\xi-y)}-q e^{\eta \lambda_{2}(\xi-y)}\right] d y-\left(e^{\lambda_{2} \xi}-q e^{\eta \lambda_{2} \xi}\right)\right] \\
& -c^{*}\left(\lambda_{2} e^{\lambda_{2} \xi}-q \eta \lambda_{2} e^{\eta \lambda_{2} \xi}\right)+r_{2}\left(e^{\lambda_{2} \xi}-q e^{\eta \lambda_{2} \xi}\right)-r_{2} e^{\lambda_{2} \xi}\left[e^{\lambda_{2} \xi}+2 b_{2} e^{\lambda_{2} \xi}\right] \\
= & -q e^{\eta \lambda_{2} \xi}\left\{d_{2}\left[\int_{\mathbb{R}} J_{2}(y) e^{\eta \lambda_{2} y} d y-1\right]-c^{*} \eta \lambda_{2}+r_{2}\right\}-r_{2} e^{\lambda_{2} \xi}\left[e^{\lambda_{2} \xi}+2 b_{2} e^{\lambda_{2} \xi}\right] \\
\geq & 0, \quad \xi<0 .
\end{aligned}
$$

By Lemma 2.4 and a discussion similar to (3.1), we complete the proof. 
Theorem 3.3 If $c^{*}=c_{2}^{*}>c_{1}^{*}$. Further suppose that $k_{2}(y)$ admits compact support. Then (2.1) with $c=c^{*}$ has a positive solution $\left(\phi_{1}(\xi), \phi_{2}(\xi)\right)$ such that

$$
0<\phi_{1}(\xi)<1,0<\phi_{2}(\xi)<1+c_{2}, \xi \in \mathbb{R}, \quad \lim _{\xi \rightarrow-\infty}\left(\phi_{1}(\xi), \phi_{2}(\xi)\right)=(0,0)
$$

and

$$
\phi_{1}(\xi) \sim \mathcal{O}\left(e^{\lambda_{1} \xi}\right), \quad \phi_{2}(\xi) \sim \mathcal{O}\left(-\xi e^{\lambda_{2}^{*} \xi}\right), \quad \xi \rightarrow-\infty
$$

Proof Under the assumption, we see that

$$
d_{2} \int_{\mathbb{R}} J_{2}(y) y e^{\lambda_{2}^{*} y} d y=c^{*}
$$

by Lemma 2.3. Let $S>0$ be a constant such that $k_{2}(y)=0,|y|>S$. Select a constant $\eta>1$ such that

$$
\lambda_{2}^{*} / 2+\lambda_{1}-\eta \lambda_{1}>0, \quad \Theta_{1}\left(\eta \lambda_{1}, c^{*}\right)<0 .
$$

Let $L>1$ be large enough such that

$$
-L \xi e^{\lambda_{2}^{*} \xi}=1+c_{2}
$$

has two real roots $\xi_{5}<\xi_{6}$ and $\xi_{6}-\xi_{5}>2 S$.

We now define

$$
\underline{\phi}_{1}(\xi)=\max \left\{e^{\lambda_{1} \xi}-q e^{\eta \lambda_{1} \xi}, 0\right\}, \quad \bar{\phi}_{1}(\xi)=\min \left\{e^{\lambda_{1} \xi}, 1\right\}
$$

and

$$
\begin{aligned}
& \underline{\phi}_{2}(\xi)= \begin{cases}(-L \xi-q \sqrt{-\xi}) e^{\lambda_{2}^{*} \xi}, & \xi<\xi_{3}, \\
0, & \xi \geq \xi_{3},\end{cases} \\
& \bar{\phi}_{2}(\xi)= \begin{cases}(-L \xi+p \sqrt{-\xi}) e^{\lambda_{2}^{*} \xi}, & \xi<\xi_{4}, \\
1+c_{2}, & \xi \geq \xi_{4},\end{cases}
\end{aligned}
$$

where $\xi_{3}=L^{2} / q^{2}$ and $\xi_{4}<\xi_{5}$ such that $\bar{\phi}_{2}(\xi)$ is continuous.

For $\bar{\phi}_{1}(\xi)$, the verification is similar to that in Theorem 3.1 and we omit it here. If $\bar{\phi}_{2}(\xi)=$ $1+c_{2}$, then $H_{2}\left(\bar{\phi}_{1}, \underline{\phi}_{2}, \bar{\phi}_{2}\right)(\xi) \leq 0$ such that $(2.7)$ is clear. Otherwise, let $p_{2}>0$ such that

$$
\bar{\phi}_{2}(\xi) \geq \underline{\phi}_{2}(\xi), \quad \xi \in \mathbb{R} .
$$

Thus,

$$
\begin{aligned}
& r_{2} \bar{\phi}_{2}(\xi) H_{2}\left(\bar{\phi}_{1}, \underline{\phi}_{2}, \bar{\phi}_{2}\right)(\xi) \\
& \quad=r_{2} \bar{\phi}_{2}(\xi)\left[1-\bar{\phi}_{2}(\xi)-b_{2} \int_{-\tau}^{0} \underline{\phi}_{2}\left(\xi+c^{*} s\right) d \eta_{22}(s)+c_{2} \int_{-\tau}^{0} \bar{\phi}_{1}\left(\xi+c^{*} s\right) d \eta_{21}(s)\right]
\end{aligned}
$$




$$
\begin{aligned}
& \leq r_{2} \bar{\phi}_{2}(\xi)\left[1+c_{2} \int_{-\tau}^{0} \bar{\phi}_{1}\left(\xi+c^{*} s\right) d \eta_{21}(s)\right] \\
& \leq r_{2} \bar{\phi}_{2}(\xi)\left[1+c_{2} e^{\lambda_{1} \xi}\right] \\
& =r_{2} e^{\lambda_{2}^{*} \xi}(-L \xi+p \sqrt{-\xi})\left[1+c_{2} e^{\lambda_{1} \xi}\right]
\end{aligned}
$$

and

$$
\begin{aligned}
& d_{2}\left[J_{2} * \bar{\phi}_{2}\right](\xi)-c^{*} \bar{\phi}_{2}^{\prime}(\xi)+r_{2} \bar{\phi}_{2}(\xi) H_{2}\left(\bar{\phi}_{1}, \underline{\phi}_{2}, \bar{\phi}_{2}\right)(\xi) \\
& \leq d_{2}\left\{\int_{\mathbb{R}} J_{2}(y)\left[(-L(\xi-y)+p \sqrt{-(\xi-y)}) e^{\lambda_{2}^{*}(\xi-y)}\right] d y\right. \\
& \left.-\left[(-L \xi+p \sqrt{-\xi}) e^{\lambda_{2}^{*} \xi}\right]\right\} \\
& -c^{*} \lambda_{2}^{*}(-L \xi+p \sqrt{-\xi}) e^{\lambda_{2}^{*} \xi}-c^{*}\left(-L-\frac{p}{2 \sqrt{-\xi}}\right) e^{\lambda_{2}^{*} \xi} \\
& +r_{2} e^{\lambda_{2}^{*} \xi}(-L \xi+p \sqrt{-\xi})+r_{2} c_{2} e^{\lambda_{2}^{*} \xi} e^{\lambda_{1} \xi}(-L \xi+p \sqrt{-\xi}) \\
& =-L \xi e^{\lambda_{2}^{*} \xi}\left\{d_{2}\left[\int_{\mathbb{R}} J_{2}(y) e^{-\lambda_{2}^{*} y} d y-1\right]-c^{*} \lambda_{2}^{*}+r_{2}\right\} \\
& +d_{2} L\left[\int_{\mathbb{R}} J_{2}(y) y e^{-\lambda_{2}^{*} y} d y+c^{*}\right] \\
& +d_{2} p e^{\lambda_{2}^{*} \xi}\left\{\left[\int_{\mathbb{R}} J_{2}(y) \sqrt{-(\xi-y)} e^{-\lambda_{2}^{*} y} d y-\sqrt{-\xi}\right]-c^{*} \lambda_{2}^{*} \sqrt{-\xi}+r \sqrt{-\xi}\right\} \\
& +\frac{c^{*} p}{2 \sqrt{-\xi}} e^{\lambda_{2}^{*} \xi}+r_{2} c_{2} e^{\lambda_{2}^{*} \xi} e^{\lambda_{1} \xi}(-L \xi+p \sqrt{-\xi}) \\
& =d_{2} p e^{\lambda_{2}^{*} \xi}\left\{\left[\int_{\mathbb{R}} J_{2}(y) \sqrt{-(\xi-y)} e^{-\lambda \lambda_{2}^{*} y} d y-\sqrt{-\xi}\right]-c^{*} \lambda_{2}^{*} \sqrt{-\xi}+r \sqrt{-\xi}\right\} \\
& +\frac{c^{*} p}{2 \sqrt{-\xi}} e^{\lambda_{2}^{*} \xi}+r_{2} c_{2} e^{\lambda_{2}^{*} \xi} e^{\lambda_{1} \xi}(-L \xi+p \sqrt{-\xi}) \\
& =d_{2} p e^{\lambda_{2}^{*} \xi} \int_{\mathbb{R}} J_{2}(y)[\sqrt{-(\xi-y)}-\sqrt{-\xi}] e^{-\lambda_{2}^{*} y} d y \\
& +\frac{c^{*} p}{2 \sqrt{-\xi}} e^{\lambda_{2}^{*} \xi}+r_{2} c_{2} e^{\lambda_{2}^{*} \xi} e^{\lambda_{1} \xi}(-L \xi+p \sqrt{-\xi}) \\
& =d_{2} p e^{\lambda_{2}^{*} \xi} \int_{\mathbb{R}} J_{2}(y)\left[\frac{y}{\sqrt{-(\xi-y)}+\sqrt{-\xi}}\right] e^{-\lambda_{2}^{*} y} d y \\
& +\frac{c^{*} p}{2 \sqrt{-\xi}} e^{\lambda_{2}^{*} \xi}+r_{2} c_{2} e^{\lambda_{2}^{*} \xi} e^{\lambda_{1} \xi}(-L \xi+p \sqrt{-\xi}) \\
& =d_{2} p e^{\lambda_{2}^{*} \xi} \int_{\mathbb{R}} J_{2}(y)\left[\frac{y}{\sqrt{-(\xi-y)}+\sqrt{-\xi}}-\frac{y}{2 \sqrt{-\xi}}\right] e^{-\lambda_{2}^{*} y} d y \\
& +r_{2} c_{2} e^{\lambda_{2}^{*} \xi} e^{\lambda_{1} \xi}(-L \xi+p \sqrt{-\xi}) \\
& =d_{2} p e^{\lambda_{2}^{*} \xi} \int_{\mathbb{R}} J_{2}(y) \frac{y[\sqrt{-\xi}-\sqrt{-(\xi-y)}]}{2 \sqrt{-\xi}[\sqrt{-(\xi-y)}+\sqrt{-\xi}]} e^{-\lambda_{2}^{*} y} d y \\
& +r_{2} c_{2} e^{\lambda_{2}^{*} \xi} e^{\lambda_{1} \xi}(-L \xi+p \sqrt{-\xi})
\end{aligned}
$$




$$
\begin{aligned}
= & d_{2} p e^{\lambda_{2}^{*} \xi} \int_{\mathbb{R}} J_{2}(y) \frac{-y^{2}}{2 \sqrt{-\xi}[\sqrt{-(\xi-y)}+\sqrt{-\xi}]^{2}} e^{-\lambda_{2}^{*} y} d y \\
& +r_{2} c_{2} e^{\lambda_{2}^{*} \xi} e^{\lambda_{1} \xi}(-L \xi+p \sqrt{-\xi}) \\
\leq & \frac{-d_{2} p e^{\lambda_{2}^{*} \xi}}{8(|\xi|+S)^{\frac{3}{2}}} \int_{\mathbb{R}} J_{2}(y) y^{2} e^{-\lambda_{2}^{*} y} d y+r_{2} c_{2} e^{\lambda_{2}^{*} \xi} e^{\lambda_{1} \xi}(-L \xi+p \sqrt{-\xi}) \\
\leq & 0
\end{aligned}
$$

if

$$
p \geq \frac{\max _{\xi<0}\left\{8 r_{2} c_{2}(|\xi|+S)^{\frac{3}{2}} e^{\lambda_{1} \xi}(-L \xi+p \sqrt{-\xi})\right\}}{d_{2} \int_{\mathbb{R}} J_{2}(y) y^{2} e^{-\lambda_{2}^{*} y} d y} .
$$

When $\underline{\phi}_{1}(\xi)=0$ with $\xi<\xi_{3}$, then $H_{1}\left(\underline{\phi}_{1}, \bar{\phi}_{1}, \bar{\phi}_{2}\right)(\xi)=0$ such that (2.6) is clear. Otherwise, if $\xi \geq \xi_{3}$, then $\underline{\phi}_{1}(\xi)=e^{\lambda_{1} \xi}-q e^{\eta \lambda_{1} \xi}>0$. Firstly, let $q>q_{1}>1$ such that $e^{\lambda_{1} \xi}-q e^{\eta \lambda_{1} \xi}>0$ implies $\xi<0$ and

$$
\bar{\phi}_{2}(\xi)<2 e^{\lambda_{2}^{*} \xi}, \quad \underline{\phi}_{1}(\xi) \leq \bar{\phi}_{1}(\xi) \leq e^{\lambda_{1} \xi}
$$

which is admissible once $p$ is fixed. Therefore, $q>q_{1}$ indicates

$$
\begin{aligned}
& r_{1} \underline{\phi}_{1}(\xi) H_{1}\left(\underline{\phi}_{1}, \bar{\phi}_{1}, \bar{\phi}_{2}\right)(\xi) \\
& \quad=r_{1} \underline{\phi}_{1}(\xi)\left[1-\underline{\phi}_{1}(\xi)-b_{1} \int_{-\tau}^{0} \bar{\phi}_{1}\left(\xi+c^{*} s\right) d \eta_{11}(s)-c_{1} \int_{-\tau}^{0} \bar{\phi}_{2}\left(\xi+c^{*} s\right) d \eta_{12}(s)\right] \\
& \quad \geq r_{1} \underline{\phi}_{1}(\xi)-r_{1} \underline{\phi}_{1}^{2}(\xi)-r_{1} b_{1} \underline{\phi}_{1}(\xi) \bar{\phi}_{1}(\xi)-2 r_{1} c_{1} e^{\lambda_{2}^{*} \xi} \underline{\phi}_{1}(\xi) \\
& \quad \geq r_{1} \underline{\phi}_{1}(\xi)-r_{1}\left(1+b_{1}\right) e^{2 \lambda_{1} \xi}-2 r_{1} c_{1} e^{\left(\lambda_{1}+\lambda_{2}^{*}\right) \xi} \\
& \quad=r_{1}\left(e^{\lambda_{1} \xi}-q e^{\eta \lambda_{1} \xi}\right)-r_{1}\left(1+b_{1}\right) e^{2 \lambda_{1} \xi}-2 r_{1} c_{1} e^{\left(\lambda_{1}+\lambda_{2}^{*}\right) \xi} .
\end{aligned}
$$

Moreover, (3.3) leads to

$$
\begin{aligned}
d_{1} J_{1} & \left.* \underline{\phi}_{1}\right](\xi)-c^{*} \underline{\phi}_{1}^{\prime}(\xi)+r_{1} \underline{\phi}_{1}(\xi) H_{1}\left(\underline{\phi}_{1}, \bar{\phi}_{1}, \bar{\phi}_{2}\right)(\xi) \\
\geq & d_{1}\left[\int_{\mathbb{R}} J_{1}(y) \underline{\phi}_{1}(\xi-y) d y-\underline{\phi}_{1}(\xi)\right]-c^{*} \underline{\phi}_{1}^{\prime}(\xi) \\
& +r_{1}\left(e^{\lambda_{1} \xi}-q e^{\eta \lambda_{1} \xi}\right)-r_{1}\left(1+b_{1}\right) e^{2 \lambda_{1} \xi}-2 r_{1} c_{1} e^{\left(\lambda_{1}+\lambda_{2}^{*}\right) \xi} \\
\geq & d_{1}\left[\int_{\mathbb{R}} J_{1}(y)\left[\left(e^{\lambda_{1}(\xi-y)}-q e^{\eta \lambda_{1}(\xi-y)}\right)\right] d y-\left(e^{\lambda_{1} \xi}-q e^{\eta \lambda_{1} \xi}\right)\right] \\
& -c^{*}\left(\lambda_{1} e^{\lambda_{1} \xi}-q \eta \lambda_{1} e^{\eta \lambda_{1} \xi}\right)+r_{1}\left(e^{\lambda_{1} \xi}-q e^{\eta \lambda_{1} \xi}\right) \\
& -r_{1}\left(1+b_{1}\right) e^{2 \lambda_{1} \xi}-2 r_{1} c_{1} e^{\left(\lambda_{1}+\lambda_{2}^{*}\right) \xi} \\
= & -q \Theta_{1}\left(\eta \lambda_{1}, c^{*}\right) e^{\eta \lambda_{1} \xi}-r_{1}\left(1+b_{1}\right) e^{2 \lambda_{1} \xi}-2 r_{1} c_{1} e^{\left(\lambda_{1}+\lambda_{2}^{*}\right) \xi} \\
\geq & 0
\end{aligned}
$$


provided that

$$
q>\frac{r_{1}\left(1+b_{1}\right)-2 r_{1} c_{1}}{-\Theta_{1}\left(\eta \lambda_{1}, c^{*}\right)}+q_{1}:=q_{2} .
$$

Let $q_{3} \geq q_{2}$ such that $q>q_{3}$ indicates

$$
\underline{\phi}_{2}(\xi)<\bar{\phi}_{2}(\xi), \quad \xi \in \mathbb{R},
$$

and $q>q_{3},(-L \xi-q \sqrt{-\xi})>0$, imply

$$
(-L \xi+q \sqrt{-\xi}) e^{\lambda_{2}^{*} \xi}<e^{2 \lambda_{2}^{*} \xi / 3}
$$

and so

$$
\begin{aligned}
& r_{2} \underline{\phi}_{2}(\xi) H_{2}\left(\underline{\phi}_{1}, \bar{\phi}_{2}, \underline{\phi}_{2}\right)(\xi) \\
& \quad=r_{2} \underline{\phi}_{2}(\xi)\left[1-\underline{\phi}_{2}(\xi)-b_{2} \int_{-\tau}^{0} \bar{\phi}_{2}\left(\xi+c^{*} s\right) d \eta_{22}(s)+c_{2} \int_{-\tau}^{0} \underline{\phi}_{1}\left(\xi+c^{*} s\right) d \eta_{21}(s)\right] \\
& \quad \geq r_{2} \underline{\phi}_{2}(\xi)\left[1-\underline{\phi}_{2}(\xi)-b_{2} \int_{-\tau}^{0} \bar{\phi}_{2}\left(\xi+c^{*} s\right) d \eta_{22}(s)\right] \\
& \quad \geq r_{2} \underline{\phi}_{2}(\xi)\left[1-\left(1+b_{2}\right) e^{2 \lambda_{2}^{*} \xi / 3}\right] \\
& \quad=r_{2}(-L \xi-q \sqrt{-\xi}) e^{\lambda_{2}^{*} \xi}-r_{2}\left(1+b_{2}\right) e^{4 \lambda_{2}^{*} \xi / 3} .
\end{aligned}
$$

By direct calculations, we see

$$
\begin{aligned}
d_{2}\left[J_{2}\right. & \left.* \underline{\phi}_{2}\right](\xi)-c^{*} \underline{\phi}_{2}^{\prime}(\xi)+r_{2} \underline{\phi}_{2}(\xi) H_{2}\left(\underline{\phi}_{1}, \bar{\phi}_{2}, \underline{\phi}_{2}\right)(\xi) \\
\geq & d_{2}\left[\int_{\mathbb{R}} J_{2}(y)\left[(-L(\xi-y)-q \sqrt{-(\xi-y)}) e^{\lambda_{2}^{*}(\xi-y)}\right] d y-(-L \xi-q \sqrt{-\xi}) e^{\lambda_{2}^{*} \xi}\right] \\
& +c^{*} L\left(1+\lambda_{2}^{*} \xi\right) e^{\lambda_{2}^{*} \xi}+c^{*} q\left(\lambda_{2}^{*} \sqrt{-\xi}-\frac{1}{2 \sqrt{-\xi}}\right) e^{\lambda_{2}^{*} \xi} \\
& +r_{2}(-L \xi-q \sqrt{-\xi}) e^{\lambda_{2}^{*} \xi}-r_{2}\left(1+b_{2}\right) e^{4 \lambda_{2}^{*} \xi / 3} \\
= & d_{2}\left[\int_{\mathbb{R}} J_{2}(y)(-L(\xi-y)) e^{\lambda_{2}^{*}(\xi-y)} d y+L \xi e^{\lambda_{2}^{*} \xi}\right]+c^{*} L\left(1+\lambda_{2}^{*} \xi\right) e^{\lambda_{2}^{*} \xi}-r_{2} L \xi e^{\lambda_{2}^{*} \xi} \\
& -q \sqrt{-\xi} d_{2} \int_{\mathbb{R}} J_{2}(y) e^{\lambda_{2}^{*}(\xi-y)} d y+q \sqrt{-\xi} e^{\lambda_{2}^{*} \xi} \\
& +c^{*} q \lambda_{2}^{*} \sqrt{-\xi} e^{\lambda_{2}^{*} \xi}+r_{2}(-L \xi-q \sqrt{-\xi}) e^{\lambda_{2}^{*} \xi} \\
& +d_{2} q \int_{\mathbb{R}} J_{2}(y)[\sqrt{-\xi}-\sqrt{-(\xi-y)}] e^{\lambda_{2}^{*}(\xi-y)} d y-\frac{c^{*} q}{2 \sqrt{-\xi}} e^{\lambda_{2}^{*} \xi}-r_{2}\left(1+b_{2}\right) e^{4 \lambda_{2}^{*} \xi / 3} \\
= & d_{2} q \int_{\mathbb{R}} J_{2}(y)[\sqrt{-\xi}-\sqrt{-(\xi-y)}] e^{\lambda_{2}^{*}(\xi-y)} d y-\frac{c^{*} q}{2 \sqrt{-\xi}} e^{\lambda_{2}^{*} \xi}-r_{2}\left(1+b_{2}\right) e^{4 \lambda_{2}^{*} \xi / 3} \\
= & d_{2} q \int_{\mathbb{R}} J_{2}(y)\left[\frac{-y}{\sqrt{-\xi}-\sqrt{-(\xi-y)}}\right] e^{\lambda_{2}^{*}(\xi-y)} d y-\frac{c^{*} q}{2 \sqrt{-\xi}} e^{\lambda_{2}^{*} \xi}-r_{2}\left(1+b_{2}\right) e^{4 \lambda_{2}^{*} \xi / 3} \\
= & d_{2} q \int_{\mathbb{R}} J_{2}(y)\left[\frac{y}{2 \sqrt{-\xi}}-\frac{y}{\sqrt{-\xi}+\sqrt{-(\xi-y)}}\right] e^{\lambda_{2}^{*}(\xi-y)} d y-r_{2}\left(1+b_{2}\right) e^{4 \lambda_{2}^{*} \xi / 3}
\end{aligned}
$$




$$
\begin{aligned}
& =d_{2} q \int_{\mathbb{R}} J_{2}(y)\left[\frac{y^{2}}{2 \sqrt{-\xi}[\sqrt{-\xi}+\sqrt{-(\xi-y)}]}\right] e^{\lambda_{2}^{*}(\xi-y)} d y-r_{2}\left(1+b_{2}\right) e^{4 \lambda_{2}^{*} \xi / 3} \\
& =d_{2} q \int_{\mathbb{R}} J_{2}(y) \frac{y^{2} e^{\lambda_{2}^{*}(\xi-y)}}{2 \sqrt{-\xi}[\sqrt{-\xi}+\sqrt{-(\xi-y)}]^{2}} d y-r_{2}\left(1+b_{2}\right) e^{4 \lambda_{2}^{*} \xi / 3} \\
& =e^{\lambda_{2}^{*} \xi}\left\{d_{2} q \int_{\mathbb{R}} J_{2}(y) \frac{y^{2} e^{\lambda_{2}^{*} y}}{2 \sqrt{-\xi}[\sqrt{-\xi}+\sqrt{-(\xi-y)}]^{2}} d y-r_{2}\left(1+b_{2}\right) e^{\lambda_{2}^{*} \xi / 3}\right\} \\
& \geq e^{\lambda_{2}^{*} \xi}\left\{d_{2} q \int_{\mathbb{R}} J_{2}(y) \frac{y^{2} e^{\lambda_{2}^{*} y}}{8(|\xi|+S)^{3 / 2}} d y-r_{2}\left(1+b_{2}\right) e^{\lambda_{2}^{*} \xi / 3}\right\} \\
& \geq 0
\end{aligned}
$$

if

$$
q \geq \sup _{\xi<0} \frac{8 e^{\lambda_{2}^{*} \xi / 3}(S+|\xi|)^{3 / 2} r_{2}\left(1+b_{2}\right)}{d_{2} \int_{\mathbb{R}} J_{2}(y) y^{2} e^{\lambda_{2}^{*} y} d y}+q_{4}:=q_{5}
$$

Fix $q=q_{5}$, we complete the proof by Lemma 2.4 and a discussion similar to (3.1).

Theorem 3.4 Assume that $c_{1}^{*}=c_{2}^{*}$. Further suppose that $k_{1}, k_{2}$ have compact supports. Then (2.1) with $c=c^{*}$ has a positive solution $\left(\phi_{1}(\xi), \phi_{2}(\xi)\right)$ such that

$$
0<\phi_{1}(\xi)<1,0<\phi_{2}(\xi)<1+c_{2}, \xi \in \mathbb{R}, \quad \lim _{\xi \rightarrow-\infty}\left(\phi_{1}(\xi), \phi_{2}(\xi)\right)=(0,0)
$$

and

$$
\phi_{1}(\xi) \sim \mathcal{O}\left(-\xi e^{\lambda_{1}^{*} \xi}\right), \quad \phi_{2}(\xi) \sim \mathcal{O}\left(-\xi e^{\lambda_{2}^{*} \xi}\right), \quad \xi \rightarrow-\infty
$$

Proof Using the notation in Theorems 3.2-3.3, we define

$$
\begin{aligned}
& \underline{\phi}_{1}(\xi)= \begin{cases}(-L \xi-q \sqrt{-\xi}) e^{\lambda_{1}^{*} \xi}, & \xi<\xi_{1}, \\
0, & \xi \geq \xi_{1},\end{cases} \\
& \bar{\phi}_{1}(\xi)= \begin{cases}-L \xi e^{\lambda_{1}^{*} \xi}, & \xi<\xi_{2}, \\
1, & \xi \geq \xi_{2},\end{cases}
\end{aligned}
$$

and

$$
\begin{aligned}
& \underline{\phi}_{2}(\xi)= \begin{cases}(-L \xi-q \sqrt{-\xi}) e^{\lambda_{2}^{*} \xi}, & \xi<\xi_{3}, \\
0, & \xi \geq \xi_{3},\end{cases} \\
& \bar{\phi}_{2}(\xi)= \begin{cases}(-L \xi+p \sqrt{-\xi}) e^{\lambda_{2}^{*} \xi}, & \xi<\xi_{4}, \\
1+c_{2}, & \xi \geq \xi_{4},\end{cases}
\end{aligned}
$$

where $p, q>1$ are large enough, $\xi_{1}, \xi_{2}, \xi_{3}, \xi_{4}$ are similar to above. Then we can obtain a pair of upper and lower solutions. Since the verification is similar to those in Theorems 3.2-3.3, we omit it here. 


\section{Asymptotic behavior and nonexistence of traveling wave solutions}

In the previous section, we obtain the existence of nonconstant traveling wave solutions of (1.1). In this part, we shall first consider the behavior if $\xi \rightarrow \infty$ by the idea of contracting rectangle [32] in Lin and Ruan [33]. For $s \in[0,1]$, define the continuous functions

$$
a_{1}(s)=s k_{1}, \quad b_{1}(s)=s k_{1}+(1-s)(1+\epsilon)
$$

and

$$
a_{2}(s)=(1-s)+s k_{2}, \quad b_{2}(s)=s k_{2}+(1-s)\left(1+c_{2}\right)(1+\epsilon)
$$

with $\epsilon \in(0,1)$ such that

$$
1-b_{1}(1+\epsilon)-c_{1}\left(1+c_{2}\right)(1+\epsilon)>0, \quad 1-b_{2}\left(1+c_{2}\right)(1+\epsilon)>0 .
$$

Then they satisfy

(C1) $1-a_{1}(s)-b_{1} b_{1}(s)-c_{1} b_{2}(s)>0$,

(C2) $1-a_{2}(s)-b_{2} b_{2}(s)+c_{2} a_{1}(s)>0$,

(C3) $1-b_{1}(s)-b_{1} a_{1}(s)-c_{1} a_{2}(s)<0$,

(C4) $1-b_{2}(s)-b_{2} a_{2}(s)+c_{2} b_{1}(s)<0$,

for any $s \in(0,1)$, we now verify them [34]. In (C1), we have

$$
\begin{aligned}
1- & a_{1}(s)-b_{1} b_{1}(s)-c_{1} b_{2}(s) \\
= & 1-s k_{1}-b_{1}\left[s k_{1}+(1-s)(1+\epsilon)\right] \\
& \quad-c_{1}\left[s k_{2}+(1-s)\left(1+c_{2}\right)(1+\epsilon)\right] \\
= & (1-s)\left[1-b_{1}(1+\epsilon)-c_{1}\left(1+c_{2}\right)(1+\epsilon)\right] \\
> & 0 .
\end{aligned}
$$

(C2) is true since

$$
\begin{aligned}
1 & -a_{2}(s)-b_{2} b_{2}(s)+c_{2} a_{1}(s) \\
& =1-s k_{2}-b_{2}\left[s k_{2}+(1-s)\left(1+c_{2}\right)(1+\epsilon)\right]+c_{2} s k_{1} \\
& =(1-s)\left[1-b_{2}\left(1+c_{2}\right)(1+\epsilon)\right] \\
& >0 .
\end{aligned}
$$

On (C3), we have

$$
\begin{aligned}
1 & -b_{1}(s)-b_{1} a_{1}(s)-c_{1} a_{2}(s) \\
& =1-\left[s k_{1}+(1-s)(1+\epsilon)\right]-b_{1} s k_{1}-c_{1}\left[(1-s)+s k_{2}\right] \\
& <1-\left[s k_{1}+(1-s)\right]-b_{1} s k_{1}-c_{1}\left[(1-s)+s k_{2}\right] \\
& =-c_{1}(1-s) \\
& \leq 0
\end{aligned}
$$


Finally, (C4) is true since

$$
\begin{aligned}
1- & b_{2}(s)-b_{2} a_{2}(s)+c_{2} b_{1}(s) \\
= & 1-\left[s k_{2}+(1-s)\left(1+c_{2}\right)(1+\epsilon)\right] \\
& \quad-b_{2}\left[(1-s)+s k_{2}\right]+c_{2}\left[s k_{1}+(1-s)(1+\epsilon)\right] \\
< & 1-\left[s k_{2}+(1-s)\left(1+c_{2}\right)\right] \\
& \quad-b_{2}\left[(1-s)+s k_{2}\right]+c_{2}\left[s k_{1}+(1-s)\right] \\
= & (1-s)\left[1-\left(1+c_{2}\right)-b_{2}+c_{2}\right] \\
= & -b_{2}(1-s) \\
\leq & 0 .
\end{aligned}
$$

Remark 4.1 In Pan [34], we proved the stability of positive steady state by $(\mathrm{C} 1)-(\mathrm{C} 4)$ of the corresponding kinetic system. Moreover, Faria [35] gave some sharp conditions on the general Lotka-Volterra systems with delays.

Theorem 4.2 Assume that $c \geq c^{*}$. Further suppose that $\left(\phi_{1}(\xi), \phi_{2}(\xi)\right)$ is a solution of (2.1) and satisfies

$$
0<\phi_{1}(\xi)<1,0<\phi_{2}(\xi)<1+c_{2}, \xi \in \mathbb{R}, \quad \lim _{\xi \rightarrow-\infty}\left(\phi_{1}(\xi), \phi_{2}(\xi)\right)=(0,0)
$$

If

$$
b_{1}+c_{1}\left(1+c_{2}\right)<1, \quad b_{2}\left(1+c_{2}\right)<1,
$$

then

$$
\lim _{\xi \rightarrow \infty} \phi_{i}(\xi)=k_{i}, \quad i=1,2 .
$$

Proof We first verify that

$$
\liminf _{\xi \rightarrow \infty} \phi_{i}(\xi)>0, \quad i=1,2
$$

By (4.1), we see that

$$
\begin{aligned}
& d_{1}\left[J_{1} * \phi_{1}\right](\xi)-c \phi_{1}^{\prime}(\xi)+r_{1} \phi_{1}(\xi) F_{1}\left(\phi_{1}, \phi_{2}\right)(\xi) \\
& \quad \geq d_{1}\left[J_{1} * \phi_{1}\right](\xi)-c \phi_{1}^{\prime}(\xi)+r_{1} \phi_{1}(\xi)\left[1-b_{1}-c_{1}\left(1+c_{2}\right)-\phi_{1}(\xi)\right]
\end{aligned}
$$

for any $\xi \in \mathbb{R}$. Then $u_{1}(x, t)=\phi_{1}(x+c t)$ satisfies

$$
\left\{\begin{array}{l}
\frac{\partial u_{1}(x, t)}{\partial t} \geq d_{1}\left[J_{1} * u_{1}\right](x, t)+r_{1} u_{1}(x, t)\left[1-b_{1}-c_{1}\left(1+c_{2}\right)-u_{1}(x, t)\right] \\
u_{1}(x, 0)=\phi_{1}(x)
\end{array}\right.
$$


for $x \in \mathbb{R}, t>0$. By Lemmas 2.1 and 2.2, we have

$$
\liminf _{t \rightarrow \infty} u_{1}(0, t) \geq 1-b_{1}-c_{1}\left(1+c_{2}\right)>0
$$

and so

$$
\liminf _{\xi \rightarrow \infty} \phi_{1}(\xi) \geq 1-b_{1}-c_{1}\left(1+c_{2}\right)>0
$$

by the definition of traveling wave solutions.

Similarly, we have

$$
\left\{\begin{array}{l}
\frac{\partial u_{2}(x, t)}{\partial t} \geq d_{2}\left[J_{2} * u_{2}\right](x, t)+r_{2} u_{2}(x, t)\left[1-b_{2}\left(1+c_{2}\right)-a_{2} u_{2}(x, t)\right] \\
u_{2}(x, 0)=\phi_{2}(x)
\end{array}\right.
$$

for $x \in \mathbb{R}, t>0$. Then Lemmas 2.1 and 2.2 imply that

$$
\liminf _{t \rightarrow \infty} u_{2}(0, t) \geq 1-b_{2}\left(1+c_{2}\right)>0
$$

and so

$$
\liminf _{\xi \rightarrow \infty} \phi_{2}(\xi) \geq 1-b_{2}\left(1+c_{2}\right)>0 .
$$

\section{Define}

$$
\begin{array}{ll}
\liminf _{\xi \rightarrow \infty} \phi_{1}(\xi)=\phi_{1}^{-}, & \liminf _{\xi \rightarrow \infty} \phi_{2}(\xi)=\phi_{2}^{-}, \\
\limsup _{\xi \rightarrow \infty} \phi_{1}(\xi)=\phi_{1}^{+}, & \limsup _{\xi \rightarrow \infty} \phi_{2}(\xi)=\phi_{2}^{+} .
\end{array}
$$

Then there exists $s^{\prime} \in(0,1]$ such that

$$
\begin{aligned}
& a_{1}\left(s^{\prime}\right) \leq \phi_{1}^{-} \leq \phi_{1}^{+} \leq b_{1}\left(s^{\prime}\right), \\
& a_{2}\left(s^{\prime}\right) \leq \phi_{2}^{-} \leq \phi_{2}^{+} \leq b_{2}\left(s^{\prime}\right) .
\end{aligned}
$$

Define $s=\sup s^{\prime}$. If $s=1$, then the result is true. Otherwise, $s<1$ and at least one of the following is true:

$$
a_{1}(s)=\phi_{1}^{-}, \quad \phi_{1}^{+}=b_{1}(s), \quad a_{2}(s)=\phi_{2}^{-}, \quad \phi_{2}^{+}=b_{2}(s) .
$$

If $a_{1}(s)=\phi_{1}^{-}$, then there exists $\left\{\xi_{m}\right\}_{m=1}^{\infty}$ such that

$$
\lim _{m \rightarrow \infty} \xi_{m}=\infty, \quad \lim _{m \rightarrow \infty} \phi_{1}\left(\xi_{m}\right)=a_{1}(s)
$$

and

$$
\liminf _{m \rightarrow \infty}\left[d_{1}\left[J_{1} * \phi_{1}\right]\left(\xi_{m}\right)-c \phi_{1}^{\prime}\left(\xi_{m}\right)\right] \geq 0 .
$$


By $(\mathrm{C} 1)$, we see that

$$
\begin{aligned}
& \liminf _{m \rightarrow \infty}\left[1-\phi_{1}\left(\xi_{m}\right)-b_{1} \int_{-\tau}^{0} \phi_{1}\left(\xi_{m}+c s\right) d \eta_{11}(s)-c_{1} \int_{-\tau}^{0} \phi_{2}\left(\xi_{m}+c s\right) d \eta_{12}(s)\right] \\
& \quad \geq 1-a_{1}(s)-b_{1} b_{1}(s)-c_{1} b_{2}(s) \\
& \quad>0
\end{aligned}
$$

which implies a contradiction by the definition of $\phi_{1}(\xi), \phi_{2}(\xi)$.

By a similar discussion of

$$
\phi_{1}^{+}=b_{1}(s), \quad a_{2}(s)=\phi_{2}^{-}, \quad \phi_{2}^{+}=b_{2}(s),
$$

we complete the proof.

We now present the nonexistence of (2.1) with (2.2) if $c<c^{*}$.

Theorem 4.3 If $c<c^{*}$, then there is not a positive solution of (2.1) with (2.2).

Proof Were the statement false, then, for some $c^{\prime} \in\left(0, c^{*}\right)$, there is a positive solution $\left(\phi_{1}(\xi), \phi_{2}(\xi)\right)$ of (2.1) with (2.2). Firstly, it is easy to confirm that

$$
0<\phi_{1}(\xi)<1, \quad 0<\phi_{2}(\xi)<1+c_{2}, \quad \xi \in \mathbb{R}
$$

If $c^{*}=c_{1}^{*}$, then there exists $\epsilon>0$ such that

$$
\inf _{\lambda>0} \frac{d_{1}\left[\int_{\mathbb{R}} J_{1}(y) e^{\lambda y} d y-1\right]+r_{1}(1-\epsilon)}{\lambda}>c^{\prime}
$$

Let $\xi^{\prime} \in \mathbb{R}$ such that

$$
\sup _{x \leq \xi^{\prime}}\left[b_{1} \int_{-\tau}^{0} \phi_{1}\left(x+c^{\prime} s\right) d \eta_{11}(s)+c_{1} \int_{-\tau}^{0} \phi_{2}\left(x+c^{\prime} s\right) d \eta_{12}(s)\right]=\epsilon,
$$

then

$$
d_{1}\left[J_{1} * \phi_{1}\right](\xi)-c \phi_{1}^{\prime}(\xi)+r_{1} \phi_{1}(\xi)\left[1-\epsilon-\phi_{1}(\xi)\right] \geq 0, \quad \xi \leq \xi^{\prime}
$$

Define $\inf _{x \geq \xi^{\prime}} \phi_{1}(\xi)=\underline{\underline{\phi_{1}}}$, then $\underline{\underline{\phi_{1}}}>0$ by the positivity and limit behavior. Let $M \geq 1$ such that

$$
M-1 \geq \frac{b_{1}+c_{1}\left(1+c_{2}\right)}{\underline{\underline{\phi_{1}}}}
$$

then

$$
d_{1}\left[J_{1} * \phi_{1}\right](\xi)-c \phi_{1}^{\prime}(\xi)+r_{1} \phi_{1}(\xi)\left[1-\epsilon-M \phi_{1}(\xi)\right] \geq 0, \quad \xi \in \mathbb{R}
$$


Therefore, $\phi_{1}(\xi)=\phi_{1}\left(x+c^{\prime} t\right)=u_{1}(x, t)$ satisfies

$$
\left\{\begin{array}{l}
\frac{\partial u_{1}(x, t)}{\partial t} \geq d_{1}\left[J_{1} * u_{1}\right](x, t)+r_{1} u_{1}(x, t)\left[1-\epsilon-M u_{1}(x, t)\right], \quad x \in \mathbb{R}, t>0 \\
u_{1}(x, 0)=\phi_{1}(x), \quad x \in \mathbb{R} .
\end{array}\right.
$$

By Lemma 2.1, we see that if

$$
-2 x=\left[\inf _{\lambda>0} \frac{d_{1}\left[\int_{\mathbb{R}} J_{1}(y) e^{\lambda y} d y-1\right]+r_{1}(1-\epsilon)}{\lambda}+c^{\prime}\right] t,
$$

then

$$
\liminf _{t \rightarrow \infty} u_{1}(x, t) \geq \frac{1-\epsilon}{M}>0,
$$

which also implies that $x+c^{\prime} t \rightarrow-\infty, t \rightarrow \infty$ and

$$
\lim _{\xi \rightarrow-\infty} \phi_{1}(\xi)=\limsup _{t \rightarrow \infty} u_{1}(x, t)=0,
$$

a contradiction occurs.

Similarly, we can prove the result if $c^{*}=c_{2}^{*}$. The proof is complete.

\section{Conclusion and discussion}

In this paper, we firstly show the existence and nonexistence of traveling wave solutions for all positive wave speed, and thus obtain the minimal wave speed. In [20, 21], the authors studied the existence of traveling wave solutions when $c>c^{*}$, and the traveling wave solutions decay exponentially. In this paper, if $c=c^{*}$, these traveling wave solutions do not decay exponentially, the asymptotic behavior coincides with the conclusions in [36, 37] when $b_{1}=b_{2}=c_{1}=c_{2}$. That is, for the minimal wave speed, the corresponding traveling wave solutions may have different properties. Moreover, there are also some results on the minimal wave speed of nonmonotone coupled systems with time delay, which was proved by constructing upper and lower solutions, part of recent results can be found in $\mathrm{Fu}$ [38], Lin [39] and Yang and Li [40].

In mathematical biology, the spreading speed is also an important threshold [41]. For monotone systems, see Liang and Zhao [3], Lui [4, 42], Weinberger [5], Weinberger et al. [6]. Recently, Pan [43] estimated the invasion speed of the predator in a predator-prey system, which equals the minimal invasion wave speed in Lin [44]. It is a challenging question to estimate the spreading speeds of (1.1), of which the corresponding undelayed system with classical Laplacian diffusion were studied by Lin [45], Pan [46], Wang and Zhang [47], Wang and Zhao [48].

\section{Funding}

The first author was partially supported by Scientific Research Project of High Education of Gansu Province of China (2016B-080) and Lanzhou City University (LZCU-QN20). The second author was partially supported by NSF of China (11461040, 11471149). The third author was partially supported by Natural Science Foundation of Jiangsu Province (BK20151288). 
Authors' contributions

All authors read and approved the final manuscript.

\section{Author details}

${ }^{1}$ School of Mathematics, Lanzhou City University, Lanzhou, People's Republic of China. ${ }^{2}$ School of Science, Lanzhou University of Technology, Lanzhou, People's Republic of China. ${ }^{3}$ School of Mathematical Science, Huaiyin Normal University, Huaian, People's Republic of China.

\section{Publisher's Note}

Springer Nature remains neutral with regard to jurisdictional claims in published maps and institutional affiliations.

\section{Received: 24 January 2018 Accepted: 22 March 2018 Published online: 03 April 2018}

\section{References}

1. Fang, J., Zhao, X.Q.: Bistable travelling waves for monotone semiflows with application. J. Eur. Math. Soc. 17 3678-3704 (2015)

2. Kumar, S., Kumar, D., Singh, J.: Fractional modelling arising in unidirectional propagation of long waves in dispersive media. Adv. Nonlinear Anal. 5, 383-394 (2016)

3. Liang, X., Zhao, X.Q.: Asymptotic speeds of spread and traveling waves for monotone semiflows with applications. Commun. Pure Appl. Math. 60, 1-40 (2007)

4. Lui, R.: Biological growth and spread modeled by systems of recursions. I. Mathematical theory. Math. Biosci. 93, 269-295 (1989)

5. Weinberger, H.F.: Long-time behavior of a class of biological model. SIAM J. Math. Anal. 13, 353-396 (1982)

6. Weinberger, H.F., Lewis, M.A., Li, B.: Analysis of linear determinacy for spread in cooperative models. J. Math. Biol. 45, $183-218(2002)$

7. Zhao, X.-Q.: Spatial dynamics of some evolution systems in biology. In: Du, Y., Ishii, H., Lin, W.Y. (eds.) Recent Progress on Reaction-Diffusion Systems and Viscosity Solutions, pp. 332-363. World Scientific, Singapore (2009)

8. Murray, J.D.: Mathematical Biology, II. Spatial Models and Biomedical Applications, 3rd edn. Interdisciplinary Applied Mathematics, vol. 18. Springer, New York (2003)

9. Shigesada, N., Kawasaki, K.: Biological Invasions: Theory and Practice. Oxford University Press, Oxford (1997)

10. Du, Y., Shi, J.: Some recent results on diffusive predator-prey models in spatially heterogeneous environment. In: Nonlinear Dynamics and Evolution Equations. Fields Inst. Commun., vol. 48, pp. 95-135. Am. Math. Soc., Providence (2006)

11. Ghergu, M., Radulescu, V.: A singular Gierer-Meinhardt system with different source terms. Proc. R. Soc. Edinb., Sect. A 138, 1215-1234 (2008)

12. Ghergu, M., Radulescu, V:: Turing patterns in general reaction-diffusion systems of Brusselator type. Commun. Contemp. Math. 12, 661-679 (2010)

13. Ghergu, M., Radulescu, V.: Nonlinear PDEs. Mathematical Models in Biology, Chemistry and Population Genetics. Springer Monographs in Mathematics. Springer, Heidelberg (2012)

14. Dunbar, S.R.: Travelling wave solutions of diffusive Lotka-Volterra equations. J. Math. Biol. 17, 11-32 (1983)

15. Dunbar, S.R.: Traveling wave solutions of diffusive Lotka-Volterra equations: a heteroclinic connection in $\mathbb{R}^{4}$. Trans. Am. Math. Soc. 286, 557-594 (1984)

16. Dunbar, S.R.: Traveling waves in diffusive predator-prey equations: periodic orbits and pointto-periodic heteroclic orbits. SIAM J. Appl. Math. 46, 1057-1078 (1986)

17. Bates, P.W.: On some nonlocal evolution equations arising in materials science. In: Brunner, H., Zhao, X., Zou, X. (eds.) Nonlinear Dynamics and Evolution Equations. Fields Inst. Commun., vol. 48, pp. 13-52. AMS, Providence (2006)

18. Fife, P.: Some nonclassical trends in parabolic and parabolic-like evolutions. In: Kirkilionis, M., Krömker, S., Rannacher, R., Tomi, F. (eds.) Trends in Nonlinear Analysis, pp. 153-191. Springer, Berlin (2003)

19. Hopf, L.: Introduction to Differential Equations of Physics. Dover, New York (1948)

20. Yu, Z., Yuan, R.: Travelling wave solutions in non-local convolution diffusive competitive-cooperative systems. IMA J. Appl. Math. 76, 493-513 (2011)

21. Zhang, G., Li, W.T., Lin, G.: Traveling waves in delayed predator-prey systems with nonlocal diffusion and stage structure. Math. Comput. Model. 49, 1021-1029 (2009)

22. Zhang, G., Li, W.T., Wang, Z.C.: Spreading speeds and traveling waves for nonlocal dispersal equations with degenerate monostable nonlinearity. J. Differ. Equ. 252, 5096-5124 (2012)

23. Jin, Y., Zhao, X.Q.: Spatial dynamics of a periodic population model with dispersal. Nonlinearity 22, 1167-1189 (2009)

24. Pan, S.: Traveling wave solutions in nonlocal dispersal models with nonlocal delays. J. Korean Math. Soc. 51, 703-719 (2014)

25. Ma, S.: Traveling wavefronts for delayed reaction-diffusion systems via a fixed point theorem. J. Differ. Equ. 171, 294-314 (2001)

26. Wu, J., Zou, X.: Traveling wave fronts of reaction-diffusion systems with delay. J. Dyn. Differ. Equ. 13, 651-687 (2001)

27. Huang, J., Zou, X.: Traveling wave solutions in delayed reaction diffusion systems with partial monotonicity. Acta Math. Appl. Sin. 22, 243-256 (2006)

28. Pan, S.: Traveling wave fronts of delayed non-local diffusion systems without quasimonotonicity. J. Math. Anal. Appl. 346, 415-424 (2008)

29. Pan, S., Li, W.T., Lin, G.: Travelling wave fronts in nonlocal delayed reaction-diffusion systems and applications. Z. Angew. Math. Phys. 60, 377-392 (2009)

30. Sun, Y., Li, W.T., Wang, Z.C.: Traveling waves for a nonlocal anisotropic dispersal equation with monostable nonlinearity. Nonlinear Anal. TMA 74, 814-826 (2011)

31. Wu, S., Liu, S.: Traveling waves for delayed non-local diffusion equations with crossing-monostability. Appl. Math. Comput. 217, 1435-1444 (2010) 
32. Smith, H.L.: Monotone Dynamical Systems: An Introduction to the Theory of Competitive and Cooperative Systems. AMS, Providence (1995)

33. Lin, G., Ruan, S.: Traveling wave solutions for delayed reaction-diffusion systems and applications to Lotka-Volterra competition-diffusion models with distributed delays. J. Dyn. Differ. Equ. 26, 583-605 (2014)

34. Pan, S.: Convergence and traveling wave solutions for a predator-prey system with distributed delays. Mediterr. J. Math. 14, Article ID 103 (2017)

35. Faria, T.: Sharp conditions for global stability of Lotka-Volterra systems with distributed delays. J. Differ. Equ. 246, 4391-4404 (2009)

36. Carr, J., Chmaj, A.: Uniqueness of travelling waves for nonlocal monostable equations. Proc. Am. Math. Soc. 132, 2433-2439 (2004)

37. Li, W.T., Sun, Y., Wang, Z.C.: Entire solutions in the Fisher-KPP equation with nonlocal dispersal. Nonlinear Anal., Real World Appl. 11, 2302-2313 (2010)

38. Fu, S.C.: Traveling waves for a diffusive SIR model with delay. J. Math. Anal. Appl. 435(1), 20-37 (2016)

39. Lin, G.: Minimal wave speed of competitive diffusive systems with time delays. Appl. Math. Lett. 76, 164-169 (2018)

40. Yang, F.-Y., Li, W.-T.: Traveling waves in a nonlocal dispersal SIR model with critical wave speed. J. Math. Anal. Appl. 458 , $1131-1146(2018)$

41. Aronson, D.G., Weinberger, H.F.: Nonlinear diffusion in population genetics, combustion, and nerve pulse propagation. In: Goldstein, J.A. (ed.) Partial Differential Equations and Related Topics. Lecture Notes in Mathematics, vol. 446, pp. 5-49. Springer, Berlin (1975)

42. Lui, R.: Biological growth and spread modeled by systems of recursions. II. Biological theory. Math. Biosci. 107, 255-287 (1991)

43. Pan, S.: Invasion speed of a predator-prey system. Appl. Math. Lett. 74, 46-51 (2017)

44. Lin, G.: Invasion traveling wave solutions of a predator-prey system. Nonlinear Anal. 96, 47-58 (2014)

45. Lin, G.: Spreading speeds of a Lotka-Volterra predator-prey system: the role of the predator. Nonlinear Anal. 74 , 2448-2461 (2011)

46. Pan, S.: Asymptotic spreading in a Lotka-Volterra predator-prey system. J. Math. Anal. Appl. 407, 230-236 (2013)

47. Wang, M., Zhang, Y.: Dynamics for a diffusive prey-predator model with different free boundaries. J. Differ. Equ. 264, 3527-3558 (2018)

48. Wang, M., Zhao, J.: A free boundary problem for the predator-prey model with double free boundaries. Dyn. Partial Differ. Equ. 29, 957-979 (2017)

\section{Submit your manuscript to a SpringerOpen ${ }^{\circ}$ journal and benefit from:}

- Convenient online submission

- Rigorous peer review

- Open access: articles freely available online

- High visibility within the field

- Retaining the copyright to your article

Submit your next manuscript at $\boldsymbol{\nabla}$ springeropen.com 